\title{
KAPLANSKY CLASSES AND DERIVED CATEGORIES
}

\author{
JAMES GILLESPIE
}

\begin{abstract}
We put a monoidal model category structure on the category of chain complexes of quasi-coherent sheaves over a quasi-compact and semiseparated scheme $X$. The approach generalizes and simplifies the method used by the author in [Gil04] and [Gil06] to build monoidal model structures on the category of chain complexes of modules over a ring and chain complexes of sheaves over a ringed space. Indeed, much of the paper is dedicated to showing that in any Grothendieck category $\mathcal{G}$, any nice enough class of objects $\mathcal{F}$ induces a model structure on the category $\mathrm{Ch}(\mathcal{G})$ of chain complexes. The main technical requirement on $\mathcal{F}$ is the existence of a regular cardinal $\kappa$ such that every object $F \in \mathcal{F}$ satisfies the following property: Each $\kappa$-generated subobject of $F$ is contained in another $\kappa$-generated subobject $S$ for which $S, F / S \in \mathcal{F}$. Such a class $\mathcal{F}$ is called a Kaplansky class. Kaplansky classes first appeared in [ELR02] in the context of modules over a ring $R$. We study in detail the connection between Kaplansky classes and model categories. We also find simple conditions to put on $\mathcal{F}$ which will guarantee that our model structure is monoidal. We will see that in several categories the class of flat objects form such Kaplansky classes, and hence induce monoidal model structures on the associated chain complex categories. We will also see that in any Grothendieck category $\mathcal{G}$, the class of all objects is a Kaplansky class which induces the usual (non-monoidal) injective model structure on $\mathrm{Ch}(\mathcal{G})$.
\end{abstract}

\section{INTRODUCTION}

The definition of left derived functor appearing in Cartan and Eilenberg's book depended on the existence of projective resolutions. Often however, especially in algebraic geometry, one is dealing with a category in which projective resolutions do not exist. For example, it is well-known that we do not have projective resolutions in the various types of sheaf categories.

Today we define derived functors by introducing the derived category $\mathcal{D}(\mathcal{A})$ of an abelian category $\mathcal{A}$. This "category" is obtained by first forming the associated category $\operatorname{Ch}(\mathcal{A})$ of unbounded chain complexes, and then formally inverting the homology isomorphisms. However, in general $\mathcal{D}(\mathcal{A})$ is not at all easy to understand. Even the morphism sets seem mysterious, without further analysis in each case. (The word "category" above is in quotes because it is only known in particular cases that the class of maps between two objects in $\mathcal{D}(\mathcal{A})$ actually form a set.) However, Quillen's notion of a model category, appearing in [Qui67], gave us a language and theory designed to deal with categories exactly like derived categories. That is, categories which are obtained by localizing with respect to a class of morphisms. Indeed, with a model structure on $\operatorname{Ch}(\mathcal{A})$, the morphism set $\mathcal{D}(\mathcal{A})(X, Y)$

Date: February 19, 2007.

Subject Classification: 55U35, 18G15, 18E30. 
for complexes $X, Y \in \mathcal{D}(\mathcal{A})$ will be isomorphic to the morphism set

$$
\operatorname{Ch}(\mathcal{A})(Q X, R Y) / \sim
$$

where $Q X$ and $R Y$ are some sort of resolutions of $X$ and $Y$ respectively (called "cofibrant" and "fibrant" replacements, respectively) and $\sim$ is the relation of chain homotopic maps. This alone gives us a better understanding of the derived category, not to mention the versatility that different model structures correspond to different cofibrant and fibrant replacements.

So given an abelian category $\mathcal{A}$, presumably without enough projective objects, we would like a model structure on $\operatorname{Ch}(\mathcal{A})$ that is good for defining left derived functors. In particular, when $\mathcal{A}$ has a tensor product, it gives rise to a tensor product on $\operatorname{Ch}(\mathcal{A})$ for which we would like left derived functors. In the language of model categories this means we would like to construct a monoidal model structure. We point out that there are other benefits to having a monoidal model structure which follow from the literature. For example, from [May3] it is would be automatic that $\mathcal{D}(\mathcal{A})$ is triangulated in a way that is strongly compatible with the derived tensor product. We would also automatically have the additivity property of generalized trace maps as defined in [May3]. If one is interested in studying the homotopy theory of monoids in $\operatorname{Ch}(\mathcal{A})$, or modules over a monoid, then one could use results from [SSO0].

We now summarize the layout of this paper. Our main tool used in proving the existence of various homological model structures is Theorem 4.12. The theorem basically says that a suitable class of objects $\mathcal{F}$ (described in some more detail below) in a Grothendieck category $\mathcal{G}$ gives rise to a model structure on $\mathrm{Ch}(\mathcal{G})$. We will ultimately use Theorem 2.2 of [Hov02] to prove Theorem 4.12. Hovey's theorem relates model structures to the notion of a cotorsion pair. Therefore much of the paper concerns cotorsion pairs and we give the necessary definitions in Section 2 . The reader can find much more on cotorsion pairs in [EJ01], [Hov02], and [Gil04]. In Section 3 we develop some theory on small cotorsion pairs. The word "small" was first used to describe a cotorsion pair in [Hov02]. An analogy is that small is to cotorsion pair as cofibrantly generated is to model category. The material in Section 3.1 was surely known to Hovey, but has not been written down in much detail. Proposition 3.8 of Section 3.2 greatly simplifies the problem of building a model structure from a small cotorsion pair and makes chunks of the work in [Gil04] and [Gil06] seem obsolete.

It is in Section 4 where we introduce the central notion of a Kaplansky class in a Grothendieck category $\mathcal{G}$. A Kaplansky class is a class $\mathcal{F}$ of objects in a Grothendieck category in which there exists a regular cardinal $\kappa$ such that every object $F \in \mathcal{F}$ satisfies the following property: Each $\kappa$-generated subobject of $F$ is contained in another $\kappa$-generated subobject $S$ for which $S, F / S \in \mathcal{F}$. The main idea of the paper is that a Kaplansky class satisfying the hypotheses of Theorem 4.12 immediately gives us a cotorsion pair which is nice enough to induce a model structure through Hovey's correspondence in [Hov02]. Our definition of Kaplansky class is a categorical version of the definition given by Edgar Enochs in [ELR02]. Enochs has proved the existence of flat covers and cotorsion envelopes in several algebraic categories, essentially by showing that the flat objects form a Kaplansky class.

It is not hard to see (but proved in this paper) that when a Kaplansky class $\mathcal{F}$ is also closed under direct limits, then every object of $\mathcal{F}$ is a transfinite extension of 
$\kappa$-generated objects in $\mathcal{F}$. In fact if we let $\widetilde{\mathcal{F}}$ be the class of all exact chain complex with cycles in the class $\mathcal{F}$, then we will see in Section 4 that each complex in $\widetilde{\mathcal{F}}$ is a transfinite extension of $\kappa$-generated complexes in $\widetilde{\mathcal{F}}$. The class $\widetilde{\mathcal{F}}$ will be the class of trivially cofibrant objects, and they are generated by the set of $\kappa$-generated complexes in $\widetilde{\mathcal{F}}$. The cofibrant complexes will be certain complexes constructed from $\kappa$-generated " $\mathcal{F}$-disks" and "F -spheres".

In Section 5 we show that if the Kaplansky class satisfies certain compatibilities with the tensor product, then the induced model structure is monoidal in the sense of [Hov99].

The rest of the paper deals with applications of Theorem 4.12. Our main concern for this paper is to prove the existence of a monoidal model structure on $\mathrm{Ch}(\mathrm{Qco}(X))$ for a nice scheme $X$. In Section 6 we see that the class of flat objects in $\mathrm{Q} c o(X)$ is a Kaplansky class which induces a monoidal model structure on $\mathrm{Ch}(\mathrm{Q} \operatorname{co}(X))$ when $X$ is a quasi-compact and semi-separated scheme. We call this model structure the flat model structure. Results from [AJL97] and [EE05] are essential to our construction of the flat model structure.

In Section 7 we give some more examples of homological model structures which come from Kaplansky classes. These include analogous flat model structures which the author previously constructed in [Gil04] and [Gil06]. The former is the flat model structure on $\mathrm{Ch}(R$-Mod) where $R$ is a commutative ring with identity. The latter is on $\mathrm{Ch}\left(\mathcal{O}_{X}\right.$-Mod $)$ where $\mathcal{O}_{X}$ is any ringed space. We will also see in Section 7 that in any Grothendieck category the class of all objects is trivially a Kaplansky class, which induces the usual injective model structure.

The author would like to thank Mark Hovey for always answering questions related to this work. A couple of lemmas which are entirely due to Hovey are pointed out in the text. Thankyou also to Edgar Enochs, and to Sergio Estrada and Leo Alonso Tarrío for helping me understand a few things they already knew about quasi-coherent sheaves. Finally, thankyou to Haynes Miller and the referees for their time and useful suggestions on improving the readability of this paper.

\section{Preliminaries}

Let $\mathcal{G}$ be an abelian category. A cotorsion pair is a pair of classes of objects $(\mathcal{A}, \mathcal{B})$ in $\mathcal{G}$ such that $\mathcal{A}^{\perp}=\mathcal{B}$ and $\mathcal{A}={ }^{\perp} \mathcal{B}$. Here $\mathcal{A}^{\perp}$ is the class of objects $X \in \mathcal{G}$ such that $\operatorname{Ext}^{1}(A, X)=0$ for all $A \in \mathcal{A}$, and similarly ${ }^{\perp} \mathcal{B}$ is the class of objects $X \in \mathcal{G}$ such that $\operatorname{Ext}^{1}(X, B)=0$ for all $B \in \mathcal{B}$. If we take $\mathcal{G}$ to be the category of modules over a fixed ring $R$, then there are two obvious cotorsion pairs. One is $(\mathcal{P}, \mathcal{A})$ where $\mathcal{P}$ is the class of projective $R$-modules and $\mathcal{A}$ is the class of all $R$-modules. The other is $(\mathcal{A}, \mathcal{I})$ where $\mathcal{I}$ is the class of injective $R$-modules. In fact, these two examples exist in any abelian category $\mathcal{G}$. Therefore they are sometimes called the categorical cotorsion pairs.

If the category $\mathcal{G}$ has a tensor product then there ought to be a "flat" cotorsion pair $(\mathcal{F}, \mathcal{C})$ as well. For example in the category $R$-Mod, taking $\mathcal{F}$ to be the class of all flat $R$-modules (tensor exact), we get that $\left(\mathcal{F}, \mathcal{F}^{\perp}\right)$ forms a cotorsion pair [EJ01]. The objects in the class $\mathcal{F}^{\perp}$ are the so-called cotorsion modules and $\mathcal{F}^{\perp}$ is usually denoted by $\mathcal{C}$.

A cotorsion pair $(\mathcal{A}, \mathcal{B})$ is said to have enough projectives if for any $X \in \mathcal{G}$ there is a short exact sequence $0 \rightarrow B \rightarrow A \rightarrow X \rightarrow 0$ where $B \in \mathcal{B}$ and $A \in \mathcal{A}$. We say it has enough injectives if it satisfies the dual statement. These concepts may 
be thought of as generalizations of the terms "enough projectives" and "enough injectives" which apply to a category $\mathcal{G}$. Indeed, to say that a category has enough projectives is the same as saying that the categorical cotorsion pair $(\mathcal{P}, \mathcal{A})$ has enough projectives. Similarly, a category has enough injectives precisely when the categorical cotorsion pair $(\mathcal{A}, \mathcal{I})$ has enough injectives.

If the category $\mathcal{G}$ has both enough projectives and injectives, then a cotorsion pair $(\mathcal{A}, \mathcal{B})$ has enough projectives if and only if it has enough injectives. This equivalence, due to Luigi Salce, is proved as Proposition 7.1.7 in [EJ01] for $R$ modules, but clearly holds in any abelian category $\mathcal{G}$ with enough projectives and injectives.

The flat cotorsion pair $(\mathcal{F}, \mathcal{C})$ is complete, but the proof is nontrivial. For a proof, one can see [EJ01]. The fact that $(\mathcal{F}, \mathcal{C})$ is a complete cotorsion pair settled a long standing conjecture of Edgar Enochs which asserted that every module has a flat cover. We refer the reader to [EJ01] for much more on cotorsion pairs, cotorsion modules, and Enochs' flat cover theorem.

A cotorsion pair $(\mathcal{A}, \mathcal{B})$ is said to be cogenerated by a set if there exists a set (not just a class) of objects $\mathcal{S} \subseteq \mathcal{A}$ for which $\mathcal{S}^{\perp}=\mathcal{B}$. It was shown in [ET01] that any cotorsion pair which is cogenerated by a set is complete, as long as we are in an $R$-module category. For categories that do not have enough projectives, we do not have an analogous theorem. However, if we slightly strengthen the definition of "cogenerated by a set" we do have an analogous theorem. This idea is due to Hovey and leads to the notion of a small cotorsion pair, which we explore in detail in Section 3. The problem of showing that a cotorsion pair is complete is analogous to the model category problem of showing that your morphisms satisfy the factorization axiom. Armed with this perspective one can see similarities between the proof in [ET01] to Quillen's proof of his small object argument. Hovey's proof in [Hov02] actually uses the small object argument.

Given an abelian category $\mathcal{G}$, we denote the associated chain complex category by $\mathrm{Ch}(\mathcal{G})$. A chain complex $\cdots \rightarrow X_{n+1} \stackrel{d_{n+1}}{\longrightarrow} X_{n} \stackrel{d_{n}}{\longrightarrow} X_{n-1} \rightarrow \cdots$ will be denoted by $(X, d)$ or simply $X$. We say $X$ is bounded below (above) if $X_{n}=0$ for all $n<k$ $(n>k)$ for some $k \in \mathbb{Z}$. We say it is bounded if it is bounded above and below. The nth cycle object is defined as ker $d_{n}$ and is denoted $Z_{n} X$. The nth boundary object is $\operatorname{Im} d_{n+1}$ and is denoted $B_{n} X$. The nth homology object is defined to be $Z_{n} X / B_{n} X$ and is denoted $H_{n} X$. Given an $M \in \mathcal{G}$, we let $S^{n}(M)$ denote the chain complex with all entries 0 except $M$ in degree $n$. We let $D^{n}(M)$ denote the chain complex $X$ with $X_{n}=X_{n-1}=M$ and all other entries 0 . All maps are 0 except $d_{n}=1_{M}$. Given $X$, the suspension of $X$, denoted $\Sigma X$, is the complex given by $(\Sigma X)_{n}=X_{n-1}$ and $\left(d_{\Sigma X}\right)_{n}=-d_{n}$. The complex $\Sigma(\Sigma X)$ is denoted $\Sigma^{2} X$ and inductively we define $\Sigma^{n} X$ for all $n \in \mathbb{Z}$. Finally, given two chain complexes, $X$ and $Y$, their tensor product $X \otimes Y$ is defined by $(X \otimes Y)_{n}=\bigoplus_{i+j=n} X_{i} \otimes Y_{j}$ in degree $n$. The boundary map $\delta_{n}$ is defined on the $(i, j)$-component of $\bigoplus_{i+j=n} X_{i} \otimes Y_{j}$ by $\left(d_{X}\right)_{i} \otimes 1_{Y_{j}}+(-1)^{i} 1_{X_{i}} \otimes\left(d_{Y}\right)_{j}$.

Recall that a Grothendieck category is an abelian category $\mathcal{G}$ which has a generator $G \in \mathcal{G}$ and in which direct limits are exact. Examples include any $R$-module category or the various sorts of sheaf categories. Also, if $\mathcal{G}$ is a Grothendieck category then so is the chain complex category $\mathrm{Ch}(\mathcal{G})$. One may peruse Appendices A and B to see some of the useful properties of Grothendieck categories, which give them a more "concrete" feel. For more information on Grothendieck categories 
(and locally presentable categories) we refer the reader to Chapter V of [Sten75], [AR94] and [Bor94].

The author has used the texts [Har77] and (to a lesser degree) [Lit82] as references for sheaves, schemes and quasi-coherent sheaves. Also see Appendix B of [TT90] for more advanced topics such as the definition and basic facts concerning semi-separated schemes. The author uses [Hov99] for referencing facts on model categories. Since the point of the paper is to show that we can construct various model categories we assume the reader knows what a model category is. However, very little knowledge of model categories is necessary to understand the paper. The work here rests heavily on the work in [Hov02] and [Gil04]. The first paper is a proper model category paper which lays out the basic interplay between cotorsion pairs and model categories, while the second focused exclusively on the interplay between cotorsion pairs of chain complexes and (homological) model structures. In particular we will use definitions and basic results from Section 3 of [Gil04]. There is a warning: Definition 3.11 in [Gil04] only makes sense if our category has enough projectives and injectives. This mistake actually leaves a gap in the construction of the flat model structure on $\mathrm{Ch}\left(\mathcal{O}_{X}\right.$-Mod) which appeared in the sequel [Gil06]. The mistake is easily fixed. Indeed one should replace the "hereditary" hypothesis with the assumption appearing as condition (4) in Theorem 4.12 of this paper. In this way we have avoided using the word "hereditary" at all in a category without enough projectives. In any case, we show in Section 7 that the flat model structure of [Gil06] is just another corollary of our Theorem 4.12.

The following definition is given in Section 3 of [Gil04]. For the readers convenience we give the definition again here.

Definition 2.1. Let $(\mathcal{A}, \mathcal{B})$ be a cotorsion pair in an abelian category $\mathcal{G}$. Let $X$ be a chain complex.

(1) $X$ is called an $\mathcal{A}$-complex if it is exact and $Z_{n} X \in \mathcal{A}$ for all $n$.

(2) $X$ is called a $\mathcal{B}$-complex if it is exact and $Z_{n} X \in \mathcal{B}$ for all $n$.

(3) $X$ is called a $d g-\mathcal{A}$ complex if $X_{n} \in \mathcal{A}$ for each $n$, and every $f: X \rightarrow B$ is null homotopic whenever $B$ is a $\mathcal{B}$-complex.

(4) $X$ is called a $d g-\mathcal{B}$ complex if $X_{n} \in \mathcal{B}$ for each $n$, and every $f: A \rightarrow X$ is null homotopic whenever $A$ is a $\mathcal{A}$-complex.

We denote the class of all $\mathcal{A}$-complexes by $\widetilde{\mathcal{A}}$ and the class of all dg- $\mathcal{A}$ complexes by $d g \widetilde{\mathcal{A}}$. Similarly, the class of all $\mathcal{B}$-complexes is denoted by $\widetilde{\mathcal{B}}$ and the class of dg- $\mathcal{B}$ complexes is denoted by $d g \widetilde{\mathcal{B}}$.

We end this section by giving proofs to a few lemmas which will be used again and again throughout the paper. The first two are very basic and concern generators in Grothendieck categories. We will usually use Lemmas 2.2 and 2.3 without explicit mention. The last lemma is a lifting property that the author learned from Mark Hovey through personal communication.

Lemma 2.2. Let $G$ be an object in an abelian category $\mathcal{G} . G$ is a generator for $\mathcal{G}$ if and only if given any morphism $d: C \rightarrow D, d$ is an epimorphism whenever $d_{*}$ is an epimorphism. Here $d_{*}: \mathcal{G}(G, C) \rightarrow \mathcal{G}(G, D)$ is defined by $d_{*}(t)=d t$.

Proof. Suppose $G$ is a generator and $d: C \rightarrow D$ is a morphism for which $d_{*}$ is an epimorphism. To show $d$ is an epimorphism, we will show that if $h d=0$, then $h=0$. By way of contradiction suppose $h \neq 0$. By definition of a generator, there exists 
a morphism $s: G \rightarrow D$ such that $h s \neq 0$. Now since $d_{*}$ is an epimorphism, there exists $t$ for which $d t=s$. So now $0=0 t=h d t=h s \neq 0$ which is a contradiction. So $d$ must be an epimorphism.

Next, suppose $d$ is an epimorphism whenever $d_{*}$ is an epimorphism and let $h: X \rightarrow Y$ be a nonzero map. To show $G$ is a generator we need to find a morphism $s: G \rightarrow X$ such that $h s \neq 0$. Let $k: Z \rightarrow X$ be the kernel of $h$ and by way of contradiction suppose $h s=0$ for all $s$. By the universal property of kernel, each $s$ factors through $k$. I.e., $k_{*}$ is an epimorphism. By hypothesis, $k$ is an epimorphism. But since $k$ is a kernel it is also a monomorphism and therefore $k$ is an isomorphism. This implies $h=0$

If $\mathcal{G}$ is an abelian category and $\mathcal{A}$ is a class of objects, we say that $\mathcal{G}$ has enough $\mathcal{A}$-objects if for any object $X \in \mathcal{G}$ we can find an epimorphism $A \rightarrow X$ where $A \in \mathcal{A}$.

Lemma 2.3. Let $\mathcal{G}$ be a Grothendieck category and $\mathcal{A}$ be a class of objects which is closed under coproducts. Then $\mathcal{A}$ contains a generator if and only if $\mathcal{G}$ has enough $\mathcal{A}$-objects.

Proof. First assume $\mathcal{A}$ contains a generator $G$ and that $X$ is an arbitrary object in $\mathcal{G}$. It is well-known (for example, see [Bor94]) that the canonical map

$$
\bigoplus_{f \in \operatorname{Hom}(G, X)} G \rightarrow X
$$

is an epimorphism. Since we assume $\mathcal{A}$ is closed under coproducts we are done.

Next assume $\mathcal{G}$ has enough $\mathcal{A}$-objects and let $G$ be a generator for $\mathcal{G}$. Then we can find an epimorphism $A \rightarrow G$ for which $A \in \mathcal{A}$. It is easy to check that $A$ too must be a generator for $\mathcal{G}$.

Lemma 2.4. Let $\mathcal{G}$ be any abelian category. Suppose we have short exact sequences $A \stackrel{i}{\rightarrow} B \stackrel{p}{\rightarrow} C$ and $K \stackrel{j}{\rightarrow} L \stackrel{q}{\rightarrow} M$ and a commutative diagram as shown below:

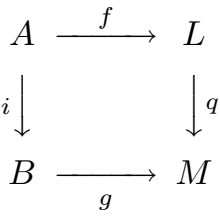

If $\operatorname{Ext}_{\mathcal{A}}^{1}(C, K)=0$ then there exists a lift $h: B \rightarrow L$ so that $h i=f$ and $q h=g$.

Proof. Consider the diagram below:

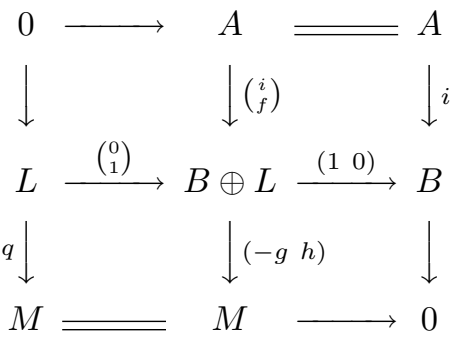

Each column forms a chain complex and so the diagram is a short exact sequence of chain complexes. The associated long exact sequence in homology leads to a short exact sequence $K \stackrel{k}{\rightarrow} T \stackrel{r}{\rightarrow} C$. 
If we let $Z$ denote the kernel of the map $(-g q): B \oplus L \rightarrow M$, then $Z$ is the pullback of the maps $q$ and $g$ as shown in the square below:

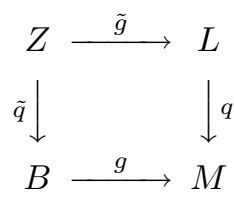

The maps $\tilde{g}$ and $\tilde{q}$ are the projections $B \oplus L \rightarrow L$ and $B \oplus L \rightarrow B$ restricted to $Z$.

Some more notation is necessary. We set $\tilde{\iota}:=\left(\begin{array}{l}i \\ f\end{array}\right): A \rightarrow Z$, and so $T=\operatorname{cok} \tilde{\iota}$. We write $\tilde{p}: Z \rightarrow T$ for the quotient map and we set $\tilde{k}:=(0 j): K \rightarrow Z$. Now one can check that the diagram below commutes, the rows and columns are exact, and the bottom right square is a pullback:

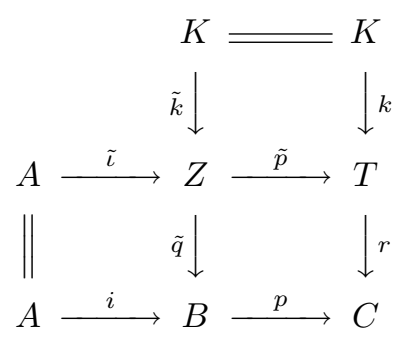

Now we can finally construct the lift. Since $\operatorname{Ext}_{\mathcal{G}}^{1}(C, K)=0$, the sequence $K \stackrel{k}{\rightarrow} T \stackrel{r}{\rightarrow} C$ splits and so we have a map $n: C \rightarrow T$ such that $r n=1_{C}$. By the pullback property, there is a unique map $\tilde{n}: B \rightarrow Z$ with $\tilde{q} \tilde{n}=1_{B}$ and $\tilde{p} \tilde{n}=$ $n p: B \rightarrow T$. We claim that the map $h:=\tilde{g} \tilde{n}: B \rightarrow L$ is a lift. Indeed, we first have $q h=q \tilde{g} \tilde{n}=g \tilde{q} \tilde{n}=g$. Next, one checks that $\tilde{p}(\tilde{\iota}-\tilde{n} i)=0$ and $\tilde{q}(\tilde{\iota}-\tilde{n} i)=0$ and so the pullback property tell us that $\tilde{n} i=\tilde{\iota}: A \rightarrow Z$. This gives us $h i=\tilde{g} \tilde{\iota}=f$ as required.

\section{SMall COTORSION PAIRS}

In the paper [Hov02] we learned a relationship between cotorsion pairs and model structures on an abelian category. In that paper Hovey defined small cotorsion pairs, which are cotorsion pairs $(\mathcal{A}, \mathcal{B})$ along with a set of "generating monomorphisms" $I$. The basic analogy is that small is to cotorsion pairs as cofibrantly generated is to model categories.

3.1. Properties of small cotorsion pairs. The results in this subsection are all known and in fact much of it can be found in Section 6 of [Hov02]. The motivation for this subsection is proving Lemmas 3.4 and 3.6. Although these were certainly known to Hovey (personal communication) they do not appear in [Hov02] and the author could not find them in the literature. Lemma 3.4 will be used in the next section while Lemma 3.6 will be used to prove that the flat model structure on chain complexes of quasi-coherent sheaves is monoidal.

Definition 3.1. Let $(\mathcal{A}, \mathcal{B})$ be a cotorsion pair in a Grothendieck category $\mathcal{G}$. Suppose $\mathcal{A}$ contains a generator $G$ for the category. The following conditions are equivalent and we say that the cotorsion pair is small if it satisfies one of these equivalent conditions: 
1) There is a set $\mathcal{S}$ which cogenerates $(\mathcal{A}, \mathcal{B})$ and for each $S \in \mathcal{S}$ there is a monomorphism $i_{S}$, with $\operatorname{cok} i_{S}=S$, satisfying the following: For all $X \in \mathcal{G}$, if $\mathcal{G}\left(i_{S}, X\right)$ is surjective for all $S \in \mathcal{S}$, then $X \in \mathcal{B}$.

2) There is a set $J$ of monomorphisms for which cok $J=\{\operatorname{cok} i: i \in J\}$ cogenerates $(\mathcal{A}, \mathcal{B})$ and which satisfies the following: For all $X \in \mathcal{G}$, if $\mathcal{G}(i, X)$ is surjective for all $i \in J$, then $X \in \mathcal{B}$.

3) There is a single monomorphism $i$ for which $\{\operatorname{cok} i\}$ cogenerates $(\mathcal{A}, \mathcal{B})$ and such that for all $X, \mathcal{G}(i, X)$ surjective implies $X \in \mathcal{B}$.

We denote by $I$ the set of monomorphisms together with the monomorphism $0 \rightarrow G$, and we call this collection the generating monomorphisms for $(\mathcal{A}, \mathcal{B})$.

The first definition is the original due to Hovey and can be found in [Hov02]. The first definition clearly implies the second definition. The second definition implies the third by looking at the direct sum $\bigoplus_{i \in I} i$. Finally the third condition clearly implies the first.

The following lemma can be found implicitly by studying Section 6 of [Hov02]. See [Hov99] for the definition of $I$-inj, $I$-cof, and $I$-cell.

Lemma 3.2. Let $(\mathcal{A}, \mathcal{B})$ be a cotorsion pair in a Grothendieck category $\mathcal{G}$. Also suppose that $\mathcal{A}$ contains a generator $G$ for the category and that the cotorsion pair is small with generating monomorphisms I. Then I-inj is the class of all epimorphisms $p$ with $\operatorname{ker} p \in \mathcal{B}$.

Proof. Say $p: X \rightarrow Y$ is in $I$-inj. Since $0 \rightarrow G$ is in $I$, there is a lift in any diagram of the form

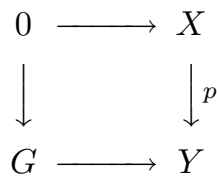

By Lemma 2.2, $p$ must be an epimorphism.

Now $\operatorname{ker} p \rightarrow 0$ can be viewed as the pullback of $p$ over the map $0 \rightarrow Y$. Since $I$-inj is closed under pullbacks we see that $\operatorname{ker} p \rightarrow 0$ is also in $I$-inj. But this is equivalent to saying $\mathcal{G}(i, \operatorname{ker} p)$ is surjective for all $i \in I$. So $\operatorname{ker} p \in \mathcal{B}$ by the definition of a set of generating monomorphisms.

On the other hand, let $p$ be an epimorphism with $\operatorname{ker} p \in \mathcal{B}$. We look for a lift in a diagram of the form

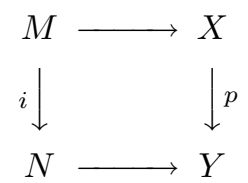

where $i \in I$. Since $\operatorname{Ext}(\operatorname{cok} i, \operatorname{ker} p)=0$, such a lift exists Lemma 2.4. This proves the Lemma.

Lemma 3.3. Let $(\mathcal{A}, \mathcal{B})$ be a cotorsion pair in a Grothendieck category $\mathcal{G}$. Also suppose that $\mathcal{A}$ contains a generator $G$ for the category and that the cotorsion pair is small with generating monomorphisms $I$. Then I-cof is the class of all monomorphisms $f$ with $\operatorname{cok} f \in \mathcal{A}$.

Proof. If $f$ is a monomorphism with $\operatorname{cok} f \in \mathcal{A}$, then $f$ is in $I$-cof by combining Lemma 3.2 and Lemma 2.4. 
Conversely, let $f: M \rightarrow N$ be in $I$-cof. Embed $M \hookrightarrow I$ in an injective. Then $(I \rightarrow 0) \in I$-inj, so there is a lift in the diagram

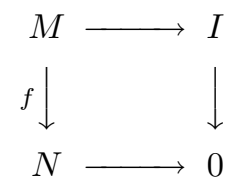

and so $f$ must be injective.

Now we wish to show that $N / M \in \mathcal{A}$. So let $B \in \mathcal{B}$ be arbitrary and embed $B$ in an injective to get a short exact sequence $0 \rightarrow B \stackrel{i}{\rightarrow} I \stackrel{j}{\rightarrow} I / B \rightarrow 0$. Since $\operatorname{Ext}^{1}(N / M, I)=0$, we will be done if we can show that any map $h: N / M \rightarrow I / B$ lifts to a map $\bar{h}: N / M \rightarrow I$ so that $h=j \bar{h}$. Notice we have a commutative diagram

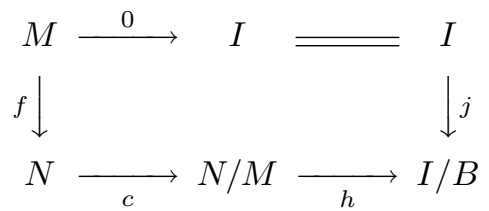

where $c: N \rightarrow N / M$ is the canonical map to the cokernel. Since $j \in I$-inj and $f \in I$-cof, there exists a lift $\psi: N \rightarrow I$ such that $\psi f=0$ and $j \psi=h c$. By the universal property of cokernel, there exists a map $\bar{h}: N / M \rightarrow I$ such that $\psi=\bar{h} c$. But now $h c=j \psi=j \bar{h} c$, and since $c$ is epi, it is right cancellable. Thus $h=j \bar{h}$.

The next lemma basically says that a set $I$ of monomorphisms (for which cok $I=$ $\mathcal{S}$ cogenerates $(\mathcal{A}, \mathcal{B})$ ) is a set of generating monomorphisms if and only if $I$-inj and $I$-cof can be classified as in the last two lemmas.

Lemma 3.4. Let $(\mathcal{A}, \mathcal{B})$ be a cotorsion pair in a Grothendieck category $\mathcal{G}$, cogenerated by a set $\mathcal{S}$. Also suppose that $\mathcal{A}$ contains a generator $G$ for the category. Suppose for each $S \in \mathcal{S}$ we are given a monomorphism $i_{S}$ with $\operatorname{cok} i_{S}=S$. Denote the set of all these $i_{S}$, together with the monomorphism $0 \rightarrow G$ by $I$. Then the following are equivalent:

1) $(\mathcal{A}, \mathcal{B})$ is a small cotorsion pair with $I$ a set of generating monomorphisms.

2) $I$-inj is the class of all epimorphisms with kernel in $\mathcal{B}$.

3) $I$-cof is the class of all monomorphisms with cokernel in $\mathcal{A}$.

Proof. (1) implies (2) is Lemma 3.2 and (1) implies (3) is Lemma 3.3. We first show (2) implies (1). So let $X \in \mathcal{G}$ and suppose $\mathcal{G}\left(i_{S}, X\right)$ is surjective for all $S \in \mathcal{S}$. This is equivalent to saying $X \rightarrow 0$ is in $I$-inj. By hypothesis $X \in \mathcal{B}$ as desired.

Next we show (3) implies (1). So let $X \in \mathcal{G}$ and suppose $\mathcal{G}\left(i_{S}, X\right)$ is surjective for all $S \in \mathcal{S}$. Again, this is equivalent to saying $X \rightarrow 0$ is in $I$-inj. We need to conclude $X \in \mathcal{B}$, so we let $A \in \mathcal{A}$ be arbitrary and argue that

$$
0 \rightarrow X \stackrel{f}{\rightarrow} Z \stackrel{g}{\rightarrow} A \rightarrow 0
$$

must split. But by hypothesis, $f \in I$-cof $=I$-inj-proj, so there is a lift in the diagram

This lift is a splitting.

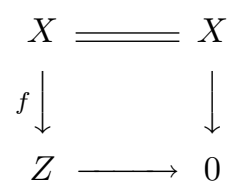


The last lemma of this section classifies the objects of $\mathcal{A}$ when $(\mathcal{A}, \mathcal{B})$ is a small cotorsion pair in which $\mathcal{A}$ contains a generator. The classification is in terms of transfinite extensions. Next, we give the definition of a transfinite extension.

Definition 3.5. A transfinite composition in an abelian category $\mathcal{G}$ is a map of the form $X_{0} \stackrel{f}{\rightarrow} \operatorname{colim} X_{\alpha}$, where $X: \lambda \rightarrow \mathcal{G}$ is a colimit-preserving functor and $\lambda$ is an ordinal. We refer to $f$ as the transfinite composition of the maps $X_{\alpha} \rightarrow X_{\alpha+1}$. If in addition, the maps $X_{\alpha} \rightarrow X_{\alpha+1}$ are all monomorphisms with cokernel in some class $\mathcal{D}$, then we refer to $f: X_{0} \rightarrow \operatorname{colim} X_{\alpha}$ as a transfinite extension of $X_{0}$ by $\mathcal{D}$. If $X_{0} \in \mathcal{D}$ as well, we just refer to colim $X_{\alpha}$ as a transfinite extension of $\mathcal{D}$.

It may be helpful to think of a transfinite extension of some class $\mathcal{D}$ in the following way. For simplicity let $\mathcal{G}$ be the category of $R$-modules. Let $\lambda$ be a limit ordinal and let $\left(M_{\alpha}\right)_{\alpha<\lambda}$ be a family of submodules of a module $M$. We call the family a continuous chain of submodules if $M_{\alpha} \subseteq M_{\alpha+1}$ for all $\alpha<\lambda$ and if $M_{\beta}=\bigcup_{\alpha<\beta} M_{\beta}$ whenever $\beta<\lambda$ is a limit ordinal. Clearly, $M$ is the union of a continuous chain of submodules $\left(M_{\alpha}\right)_{\alpha<\lambda}$ iff $M_{0} \subseteq M$ is a transfinite composition of the maps $M_{\alpha} \subseteq M_{\alpha+1}$. If $M_{0} \in \mathcal{D}$ and $M_{\alpha+1} / M_{\alpha} \in \mathcal{D}$, where $\mathcal{D}$ is some class of modules, then $M$ is a transfinite extension of $\mathcal{D}$.

Lemma 3.6. Let $(\mathcal{A}, \mathcal{B})$ be a cotorsion pair in a Grothendieck category $\mathcal{G}$. Also suppose that $\mathcal{A}$ contains a generator $G$ for the category and that the cotorsion pair is small with cogenerating set $\mathcal{S}$ and generating monomorphisms $I$. Then every object $A \in \mathcal{A}$ is a retract of a transfinite extension of objects in $\mathcal{S} \cup\{G\}$. In particular, $\mathcal{A}$ is the smallest class containing $\mathcal{S} \cup\{G\}$ and closed under transfinite extensions and retracts (summands).

Proof. Let $A \in \mathcal{A}$. Then $0 \rightarrow A$ is in $I$-cof by Lemma 3.3. By Corollary 2.1.15 of [Hov99] we see $0 \rightarrow A$ is a retract of a map $0 \rightarrow Y$ in $I$-cell, by a map which fixes 0 . That is, $A$ is a retract of $Y$. But if $0 \rightarrow Y$ is in $I$-cell, then $Y$ is a transfinite extension of the cokernels of maps in $I$. (This follows right from the definition of $I$-cell and the fact that cokernels are unchanged when pushing out over a monomorphism.) So $A$ is a retract of a transfinite extension of $\mathcal{S} \cup\{G\}$.

Say $\mathcal{W}$ is the smallest class containing $\mathcal{S} \cup\{G\}$ and is closed under transfinite extensions and retracts. Now $\mathcal{A}$ contains $\mathcal{S} \cup\{G\}$ and we know from Lemma 6.2 of [Hov02] that the left side of a cotorsion pair is always closed under transfinite extensions and retracts. So $\mathcal{A} \supseteq \mathcal{W}$. Conversely, from the last paragraph it is clear that $\mathcal{A} \subseteq \mathcal{W}$.

3.2. An associated cotorsion pair of chain complexes is small. We continue to let $(\mathcal{A}, \mathcal{B})$ represent a small cotorsion pair in a Grothendieck category $\mathcal{G}$ which has a generator $G \in \mathcal{A}$. By Corollary 3.8 of [Gil04], we have induced cotorsion pairs $(d g \widetilde{\mathcal{A}}, \widetilde{\mathcal{B}})$ and $(\widetilde{\mathcal{A}}, d g \widetilde{\mathcal{B}})$ of chain complexes, where the classes $d g \widetilde{\mathcal{A}}, \widetilde{\mathcal{B}}, \widetilde{\mathcal{A}}$ and $d g \widetilde{\mathcal{B}}$ are those defined in Section 2 above. We now show $(d g \widetilde{\mathcal{A}}, \widetilde{\mathcal{B}})$ is small whenever $(\mathcal{A}, \mathcal{B})$ is small. The author doesn't know of a corresponding theorem for the cotorsion pair $(\widetilde{\mathcal{A}}, d g \widetilde{\mathcal{B}})$ without making further assumptions on the class $\mathcal{A}$. This is the subject of Section 4.

Lemma 3.7. Let $X$ be a chain complex in an abelian category $\mathcal{G}$ with generator $G$. If any chain map $f: S^{n}(G) \rightarrow X$ extends to $D^{n+1}(G)$, then $X$ is exact. 
Proof. Let $n$ be an arbitrary integer. By Lemma 2.2, showing exactness in degree $n$ requires showing that any morphism $f: G \rightarrow Z_{n} X$ lifts over $d: X_{n+1} \rightarrow Z_{n} X$. But it is easy to see that this is the same as showing that the induced chain map $\hat{f}: S^{n}(G) \rightarrow X$ extends to a morphism $D^{n+1}(G) \rightarrow X$.

Proposition 3.8. Let $(\mathcal{A}, \mathcal{B})$ be a cotorsion pair in a Grothendieck category $\mathcal{G}$ which has a generator $G \in \mathcal{A}$. If $(\mathcal{A}, \mathcal{B})$ is cogenerated by a set $\left\{A_{i}\right\}_{i \in I}$, then the induced cotorsion pair $(d g \widetilde{\mathcal{A}}, \widetilde{\mathcal{B}})$ is cogenerated by the set

$$
S=\left\{S^{n}(G) \mid n \in \mathbb{Z}\right\} \cup\left\{S^{n}\left(A_{i}\right) \mid n \in \mathbb{Z}, i \in I\right\} .
$$

Furthermore, suppose $(\mathcal{A}, \mathcal{B})$ is small with generating monomorphisms the map $0 \rightarrow G$ together with monomorphisms $k_{i}$ as below (one for each $i \in I$ ):

$$
0 \rightarrow Y_{i} \stackrel{k_{i}}{\longrightarrow} Z_{i} \rightarrow A_{i} \rightarrow 0 .
$$

Then $(d g \widetilde{\mathcal{A}}, \widetilde{\mathcal{B}})$ is small with generating monomorphisms the set

$$
I=\left\{0 \rightarrow D^{n}(G)\right\} \cup\left\{S^{n-1}(G) \rightarrow D^{n}(G)\right\} \cup\left\{S^{n}\left(Y_{i}\right) \stackrel{S^{n}\left(k_{i}\right)}{\longrightarrow} S^{n}\left(Z_{i}\right)\right\} .
$$

Proof. Clearly $S \subseteq d g \widetilde{\mathcal{A}}$, so we have $S^{\perp} \supseteq(d g \widetilde{\mathcal{A}})^{\perp}=\widetilde{\mathcal{B}}$. Conversely if $X \in S^{\perp}$, then $0=\operatorname{Ext}_{\mathrm{Ch}(\mathcal{G})}^{1}\left(S^{n}\left(A_{i}\right), X\right)$ for all $i \in I$. But $\operatorname{Ext}_{\mathrm{Ch}(\mathcal{G})}^{1}\left(S^{n}\left(A_{i}\right), X\right) \cong \operatorname{Ext}_{\mathcal{G}}^{1}\left(A_{i}, Z_{n} X\right)$ (Lemma 3.1 in [Gil04]). So $\operatorname{Ext}_{\mathcal{G}}^{1}\left(A_{i}, Z_{n} X\right)=0$ which implies $Z_{n} X \in \mathcal{B}$ since the set $\left\{A_{i}\right\}$ cogenerates the cotorsion theory.

Next we want to show that $X$ is exact. Consider the short exact sequence

$$
0 \rightarrow S^{n-1}(G) \rightarrow D^{n}(G) \rightarrow S^{n}(G) \rightarrow 0 .
$$

It induces an exact sequence of abelian groups

$$
\operatorname{Hom}_{\mathrm{Ch}(\mathcal{G})}\left(D^{n}(G), X\right) \rightarrow \operatorname{Hom}_{\mathrm{Ch}(\mathcal{G})}\left(S^{n-1}(G), X\right) \rightarrow \operatorname{Ext}_{\mathrm{Ch}(\mathcal{G})}\left(S^{n}(G), X\right) .
$$

But again Lemma 3.1 of [Gil04] gives us $\operatorname{Ext}_{\mathrm{Ch}(\mathcal{G})}\left(S^{n}(G), X\right) \cong \operatorname{Ext}_{\mathcal{G}}\left(G, Z_{n} X\right)$ and this last group equals 0 by the last paragraph. Therefore Lemma 3.7 tells us $X$ is exact. Since $X$ is exact and has cycles in $\mathcal{B}$, we see that $X \in \widetilde{\mathcal{B}}$. So $S^{\perp}=\widetilde{\mathcal{B}}$. This shows that $\mathcal{S}$ cogenerates the cotorsion pair $(d g \widetilde{\mathcal{A}}, \widetilde{\mathcal{B}})$.

Next we prove the statement about smallness. First note that since $G$ generates $\mathcal{G}$, the complexes $D^{n}(G)$ generate $\operatorname{Ch}(\mathcal{G})$. Also $D^{n}(G) \in d g \widetilde{\mathcal{A}}$, and so $d g \widetilde{\mathcal{A}}$ contains the generators $\left\{D^{n}(G)\right\}$. Now let $X$ be any chain complex. We wish to show that "extending through monomorphisms in $I$ " implies $X \in \widetilde{\mathcal{B}}$. But again, if morphisms $S^{n-1}(G) \rightarrow X$ can extend over $D^{n}(G)$, then $X$ must be exact by Lemma 3.7. Next let $Z_{n} X$ be a cycle. Any map $Y_{i} \rightarrow Z_{n} X$ determines a morphism $S^{n}\left(Y_{i}\right) \rightarrow X$, which we assume extends over $S^{n}\left(k_{i}\right)$ to a map $S^{n}\left(Z_{i}\right) \rightarrow X$. Thus any map $Y_{i} \rightarrow Z_{n} X$ extends over $k_{i}$ to a map $Z_{i} \rightarrow Z_{n} X$. By hypothesis this implies $Z_{n} X \in \mathcal{B}$.

As mentioned at the beginning of this subsection, we know from Corollary 3.8 of [Gil04] that if $\mathcal{A}$ contains a generator for $\mathcal{G}$ and $(\mathcal{A}, \mathcal{B})$ is a cotorsion pair, then we have two induced cotorsion pairs on $\operatorname{Ch}(\mathcal{A})$. We denote them by $(d g \widetilde{\mathcal{A}}, \widetilde{\mathcal{B}})$ and $(\widetilde{\mathcal{A}}, d g \widetilde{\mathcal{B}})$ as in [Gil04]. We will see in the proof of Theorem 4.12 that a crucial step in building a model category structure on $\operatorname{Ch}(\mathcal{A})$ is showing that these induced cotorsion pairs are compatible. This means $d g \widetilde{\mathcal{A}} \cap \mathcal{E}=\widetilde{\mathcal{A}}$ and $d g \widetilde{\mathcal{B}} \cap \mathcal{E}=\widetilde{\mathcal{B}}$. Condition (4) of Corollary 3.9 below will be used at that time to guarantee that the induced cotorsion pairs are compatible. 
Corollary 3.9. Let $(\mathcal{A}, \mathcal{B})$ be a small cotorsion pair in a Grothendieck category $\mathcal{G}$. Assume $\mathcal{A}$ contains a generator for $\mathcal{G}$. Then the following are equivalent:

(1) The induced cotorsion pairs $(d g \widetilde{\mathcal{A}}, \widetilde{\mathcal{B}})$ and $(\widetilde{\mathcal{A}}, d g \widetilde{\mathcal{B}})$ are compatible.

(2) $\mathcal{A}$ is resolving and $\mathcal{B}$ is coresolving. That is, $\mathcal{A}$ is closed under taking kernels of epimorphisms and $\mathcal{B}$ is closed under taking cokernels of monomorphisms.

(3) $\operatorname{Ext}_{\mathcal{A}}^{n}(A, B)=0$ for any $n>0$ and any $A \in \mathcal{A}$ and $B \in \mathcal{B}$.

(4) $\widetilde{\mathcal{A}}=d g \widetilde{\mathcal{A}} \cap \mathcal{E}$

Proof. First we show (1) implies (2). Say we are given a short exact sequence $0 \rightarrow X \rightarrow A_{0} \rightarrow A \rightarrow 0$ where $A_{0}$ and $A$ are in the class $\mathcal{A}$. Since $\mathcal{A}$ contains a generator we can complete the short exact sequence to obtain an " $\mathcal{A}$-resolution" $\cdots \rightarrow A_{2} \rightarrow A_{1} \rightarrow A_{0} \rightarrow A \rightarrow 0$. Since this resolution is a bounded below complex with objects in $\mathcal{A}$, it is in $d g \widetilde{\mathcal{A}}$ by Lemma 3.4 of [Gil04]. But it is also in $\mathcal{E}$. Since $d g \widetilde{\mathcal{A}} \cap \mathcal{E}=\widetilde{\mathcal{A}}$, we see that the resolution is in $\widetilde{\mathcal{A}}$. Therefore $X \in \mathcal{A}$ by the definition of the class $\widetilde{\mathcal{A}}$. This shows $\mathcal{A}$ is resolving. The dual shows that $\mathcal{B}$ is coresolving.

Next we show (2) implies (3). We will prove by induction that $\operatorname{Ext}^{n}(A, B)=0$ for all $n>0, A \in \mathcal{A}$ and $B \in \mathcal{B}$. Obviously $\operatorname{Ext}^{1}(A, B)=0$ for any $A \in \mathcal{A}$ and $\mathcal{B}$. Now suppose $k>0$ and that $\operatorname{Ext}^{k}(A, B)=0$ for any $A \in \mathcal{A}$ and $B \in \mathcal{B}$. We now let $A \in \mathcal{A}$ and $B \in \mathcal{B}$ be arbitrary but fixed and wish to argue $\operatorname{Ext}^{k+1}(A, B)=0$. We start by embedding $B$ inside an injective, $0 \rightarrow B \rightarrow I \rightarrow B^{\prime} \rightarrow 0$. Note that $I \in \mathcal{B}$ and so by hypothesis $B^{\prime} \in \mathcal{B}$. We now apply the functor $\operatorname{Hom}(A,-)$ to get the long exact sequence $\cdots \rightarrow \operatorname{Ext}^{k}\left(A, B^{\prime}\right) \rightarrow \operatorname{Ext}^{k+1}(A, B) \rightarrow \operatorname{Ext}^{k+1}(A, I) \rightarrow \cdots$. By the induction hypothesis $\operatorname{Ext}^{k}\left(A, B^{\prime}\right)=0$ and since $I$ is injective $\operatorname{Ext}^{k+1}(A, I)=0$. It follows that $\operatorname{Ext}^{k+1}(A, B)=0$. Therefore (2) implies (3).

It is easy to see that (3) implies (2), so we have that (2) and (3) are equivalent.

Now we show (2) implies (4). First use Lemma 3.10 of [Gil04] to see that $\widetilde{\mathcal{A}} \subset$ $d g \widetilde{\mathcal{A}} \cap \mathcal{E}$. Next, using only the coresolving hypothesis in (2), we can perform the (dual of the) argument in the proof of Theorem 3.12 of [Gil04] to conclude $\widetilde{\mathcal{A}} \supset d g \widetilde{\mathcal{A}} \cap \mathcal{E}$. So $\widetilde{\mathcal{A}}=d g \widetilde{\mathcal{A}} \cap \mathcal{E}$.

It is left to show that (4) implies (1) and this amounts to showing $\widetilde{\mathcal{B}}=d g \widetilde{\mathcal{B}} \cap \mathcal{E}$. But by Proposition 3.8 we see that $(d g \widetilde{\mathcal{A}}, \widetilde{\mathcal{B}})$ is a small cotorsion pair. By Corollary 6.6 of [Hov02] we get that $(d g \widetilde{\mathcal{A}}, \widetilde{\mathcal{B}})$ has enough injectives. Finally Lemma 3.14 of [Gil04] tells us $\widetilde{\mathcal{B}}=d g \widetilde{\mathcal{B}} \cap \mathcal{E}$.

\section{Locally COGEnerated Classes and Kaplansky CLASSES}

Throughout this section we again assume that $\mathcal{G}$ is a Grothendieck category. In each of the categories $R$-Mod, $\mathcal{O}_{X}$-Mod and $\mathrm{Qco}(X)$, the class of flat objects satisfies an important property. In a loose sense, every flat object is "built" from "smaller" flat objects. "Built" in this case means "is a transfinite extension of" and by "smaller" we essentially mean "of smaller cardinality". We axiomatize this to get the concept of a locally cogenerated class. We will prove that a locally cogenerated class $\mathcal{F}$ containing a generator and closed under transfinite extensions and retracts gives rise to a small cotorsion pair $(\mathcal{F}, \mathcal{C})$. However, in light of Section 3.2, to get the flat model structure (on either $R$-Mod, $\mathcal{O}_{X}$-Mod or $\mathrm{Qco}(X)$ ) we really need the induced cotorsion pair $(\widetilde{\mathcal{F}}, d g \widetilde{\mathcal{C}})$ to be small. We will do this by showing $\widetilde{\mathcal{F}}$ is a locally cogenerated class. Doing so will lead us to the notion of a Kaplansky class, which is a slight strengthening of a locally cogenerated class. The author got the 
term "Kaplansky class" from Edgar Enochs whose work (with several coauthors) on proving the existence of flat covers in categories such as $R$-Mod, $\mathcal{O}_{X}$-Mod and $\mathrm{Qco}(X)$ is closely related to the idea of a Kaplansky class. See [BBE01] , [ELR02] , [EE05], [EEGO], and [EO01]. In each category mentioned above, Enochs and coauthors have essentially shown that the class of flat objects form a Kaplansky class. So as a result of Theorem 4.12 we then have an induced (flat) model structure on the associated chain complex category. We explain in section 6 using results of [EE05] why the class of flat objects in $\mathrm{Qco}(X)$ is a Kaplansky class.

The reader should now skim through Appendix A since many definitions and theorems we use in this section can be found there.

\subsection{Locally cogenerated classes.}

Definition 4.1. Let $\kappa$ be a regular cardinal. Given a class $\mathcal{F}$ of objects in $\mathcal{G}$, we say $\mathcal{F}$ is locally $\kappa$-cogenerated if for every $0 \neq F \in \mathcal{F}$, there exists $0 \neq S \subseteq F$ with $S \in \operatorname{Gen}_{\kappa} \mathcal{F}$ and $F / S \in \mathcal{F}$. We say $\mathcal{F}$ is locally cogenerated if it is locally $\kappa$-cogenerated for some regular cardinal $\kappa$.

Fact 4.2. Let $\kappa^{\prime}$ and $\kappa$ be regular cardinals with $\kappa^{\prime} \geq \kappa$. It follows from Fact A.2 that if $\mathcal{F}$ is locally $\kappa$-cogenerated, then $\mathcal{F}$ is locally $\kappa^{\prime}$-cogenerated.

Lemma 4.3. Suppose the class $\mathcal{F}$ is locally $\kappa$-cogenerated and is closed under direct limits. Then given a monomorphism $f: A \hookrightarrow B$ with $B / A \in \mathcal{F}$, we may write $B$ as a transfinite extension of $A$ by objects in $\mathbf{G e n}_{\kappa} \mathcal{F}$. In particular, every object of $\mathcal{F}$ is a transfinite extension of $\mathbf{G e n}_{\kappa} \mathcal{F}$

Proof. Set $X_{0}=A$. Now use the definition of locally $\kappa$-cogenerated to find $0 \neq$ $X_{1} / X_{0} \subseteq B / X_{0}$ with $X_{1} / X_{0} \in \mathbf{G e n}_{\kappa} \mathcal{F}$ and $B / X_{1} \in \mathcal{F}$. Continue, by transfinite induction, setting $X_{\gamma}=\bigcup_{\alpha<\gamma} X_{\alpha}$ for limit ordinals $\gamma$. Since $\mathcal{F}$ is closed under direct limits we get that $B / X_{\gamma} \in \mathcal{F}$, and we may continue the induction. Eventually, this process must terminate (since $B$ has only a set of subobjects by Fact A.11), and we get $B=\bigcup_{\alpha<\lambda} X_{\alpha}$ for some ordinal $\lambda$. This says $B$ is a transfinite extension of $A$ by objects in $\operatorname{Gen}_{\kappa} \mathcal{F}$ since $X_{0}=A$ and $X_{\alpha+1} / X_{\alpha} \in \mathbf{G e n}_{\kappa} \mathcal{F}$.

The author learned the following Lemma from Mark Hovey. It is a generalization of Lemma V.3.3 from [Sten75].

Lemma 4.4. Suppose $\mathcal{G}$ is locally $\kappa$-generated. Given an epimorphism $g: X \rightarrow Y$ where $Y$ is $\kappa$-generated, there exists a $\kappa$-generated subobject $X^{\prime} \subseteq X$ for which $g_{\mid X^{\prime}}: X^{\prime} \rightarrow Y$ is an epimorphism.

Proof. Since $\mathcal{G}$ is locally $\kappa$-generated, we may write $X=\sum_{i \in I} X_{i}$ as a $\kappa$-filtered union of $\kappa$-generated subobjects of $X$. Since $g$ is an epimorphism, $Y=\sum_{i \in I} g\left(X_{i}\right)$, and this too is a $\kappa$-filtered union. Now we must have $Y=g\left(X_{i}\right)$ for some $i \in I$ by Fact A.5. So $g_{\mid X_{i}}: X_{i} \rightarrow Y$ is an epimorphism.

Definition 4.5. Suppose $\mathcal{F}$ is locally cogenerated class in $\mathcal{G}$. By Facts A.8 and 4.2 we can choose a regular cardinal $\kappa$ with each of the following properties: (1) $\mathcal{G}$ is locally $\kappa$-presentable (hence locally $\kappa$-generated too) and $(2) \mathcal{F}$ is locally $\kappa$ cogenerated. Having chosen such a $\kappa$, we define $I$ to be the set of all (representatives of isomorphism classes of) monomorphisms $A \hookrightarrow B$ for which $B \in \mathbf{G e n}_{\kappa} \mathcal{G}$ and $B / A \in \mathcal{F}$. Furthermore, if $\mathcal{F}$ contains a generator $G$, then we also assume we have chosen $\kappa$ large enough so that (3) $G$ is $\kappa$-presentable. 
Note that by Fact A.4, if $(A \hookrightarrow B) \in I$ then we have $B / A \in \mathbf{G e n}_{\kappa} \mathcal{F}$. Also, $(0 \hookrightarrow B) \in I$ iff $B \in \mathbf{G e n}_{\kappa} \mathcal{F}$.

Lemma 4.6. Let $\mathcal{F}$ be a locally cogenerated class in $\mathcal{G}$ which is closed under direct limits. If $A \hookrightarrow B$ is a monomorphism with $B / A \in \mathcal{F}$ then $f: A \rightarrow B \in I$-cell. The converse holds if we also assume that $\mathcal{F}$ is closed under extensions.

Proof. We first need to show that any $f: A \hookrightarrow B$ with $B / A \in \mathcal{F}$ is in $I$-cell. Using Lemma 4.3, we may write $B=\bigcup_{\alpha<\lambda} X_{\alpha}$ in such a way that $X_{0}=A$ and $X_{\alpha+1} / X_{\alpha} \in \mathbf{G e n}_{\kappa} \mathcal{F}$. So $f$ is the composition of the $\lambda$-sequence

$$
X_{0}=A \hookrightarrow X_{1} \hookrightarrow X_{2} \cdots \rightarrow X_{\alpha} \hookrightarrow X_{\alpha+1} \rightarrow \cdots
$$

where each $X_{\alpha} \hookrightarrow X_{\alpha+1}$ satisfies $X_{\alpha+1} / X_{\alpha} \in \mathbf{G e n}_{\kappa} \mathcal{F}$. But since $X_{\alpha+1} / X_{\alpha}$ is $\kappa$ generated we can, by Lemma 4.4 , find $X_{\alpha+1}^{\prime} \subseteq X_{\alpha+1}$ such that $X_{\alpha+1}^{\prime}$ is $\kappa$-generated and $X_{\alpha+1}^{\prime} \rightarrow X_{\alpha+1} / X_{\alpha}$ is surjective. Then note $X_{\alpha+1}=X_{\alpha+1}^{\prime}+X_{\alpha}$. Now we have the following commutative diagram, where the rows are short exact sequences and the left square is a pushout (and pullback):

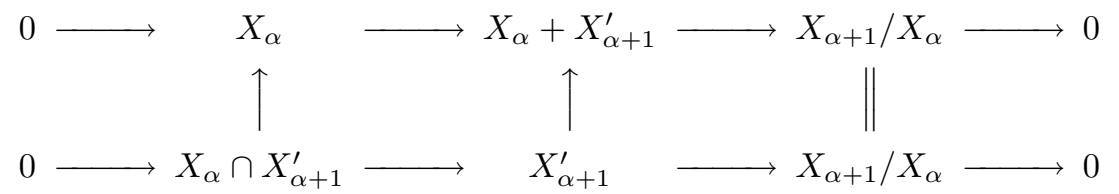

Now $X_{\alpha} \cap X_{\alpha+1}^{\prime} \hookrightarrow X_{\alpha+1}^{\prime}$ is in $I$ by definition. This shows $f$ is in $I$-cell, since $f$ is a transfinite composition of pushouts of maps in $I$.

To prove the converse, we actually only need to assume that $\mathcal{F}$ is closed under transfinite extensions. (We don't even need the locally cogenerated hypothesis for this direction.) Indeed suppose $f: A \rightarrow B$ is in $I$-cell, so that it is the composition of some $\lambda$-sequence $A=X_{0} \rightarrow \cdots X_{\alpha} \rightarrow X_{\alpha+1} \cdots$ where each $X_{\alpha} \rightarrow X_{\alpha+1}$ is a pushout of some $A_{\alpha} \hookrightarrow A_{\alpha+1}$ in $I$. Since a pushout of a mono is a mono, each $X_{\alpha} \hookrightarrow X_{\alpha+1}$ must be a monomorphism. It follows that $f$, being the map from $A$ to the colimit of the $\lambda$-sequence, $B$, is also a monomorphism.

Now take the quotient of the entire diagram, by $A=X_{0}$. It gives a new cone whose base is the $\lambda$-sequence $0 \hookrightarrow X_{1} / X_{0} \hookrightarrow \cdots X_{\alpha} / X_{0} \hookrightarrow X_{\alpha+1} / X_{0} \cdots$. All maps in the new diagram are also monomorphisms and the universal property of a quotient object will show that the new cone is actually a colimit cone, with colimit $B / A$. Furthermore the new diagram shows that $B / A$ is a transfinite extension of things in $\mathcal{F}$, since each $\left(X_{\alpha+1} / X_{0}\right) /\left(X_{\alpha} / X_{0}\right) \cong X_{\alpha+1} / X_{\alpha}$ is in the class $\mathcal{F}$ as the following pushout diagram shows:

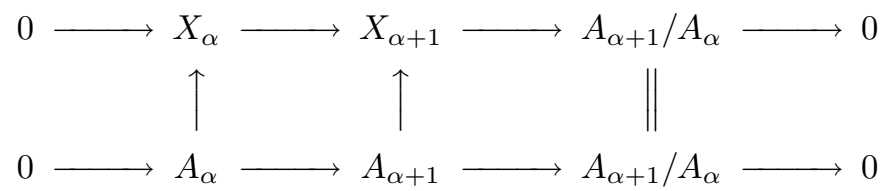

Remark. Let $\mathcal{F}$ be a locally cogenerated class in $\mathcal{G}$ which is closed under direct limits. If $F \in \mathcal{F}$ then $0 \rightarrow F \in I$-cell. The converse holds if $\mathcal{F}$ is closed under transfinite extensions. In particular, when $\mathcal{F}$ is closed under direct limits and extensions. 
Lemma 4.7. Let $\mathcal{F}$ be a locally cogenerated class which is closed under direct limits and contains a generator $G$. Then $I$-inj equals the class of all maps $p: X \rightarrow Y$ such that $p$ is surjective and $\operatorname{ker} p \in\left(\operatorname{Gen}_{\kappa} \mathcal{F}\right)^{\perp}$

Proof. Suppose $p: X \rightarrow Y$ is in $I$-inj. First we show that $p$ must be surjective. Using Lemma 2.2 this is easy: $0 \rightarrow G \in I$ and so there is a lift in any diagram of the type below.

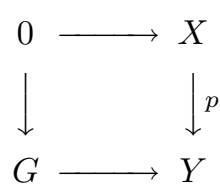

Next we show $\operatorname{ker} p \in\left(\mathbf{G e n}_{\kappa} \mathcal{F}\right)^{\perp}$. Let $F \in \mathbf{G e n}_{\kappa} \mathcal{F}$ be arbitrary. We want to show that any extension $0 \rightarrow \operatorname{ker} p \stackrel{f}{\rightarrow} Z \stackrel{g}{\rightarrow} F \rightarrow 0$ must split. But by Lemma 4.6, $f \in I$-cell $\subseteq I$-cof, so there exists a lift in the commutative diagram

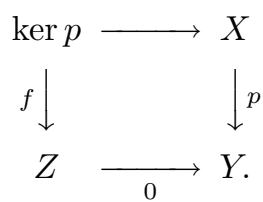

Call the lift $h$. Since $p h=0, h$ lands in $\operatorname{ker} p$ and so provides a retraction for $f$. This shows $\operatorname{ker} p \in\left(\mathbf{G e n}_{\kappa} \mathcal{F}\right)^{\perp}$.

Conversely, say $p: X \rightarrow Y$ is a surjection and $\operatorname{ker} p \in\left(\mathbf{G e n}_{\kappa} \mathcal{F}\right)^{\perp}$. Given any map $f: A \rightarrow B$ in $I$, and a commutative diagram

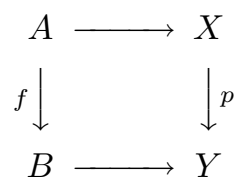

we seek a lift. But since $\operatorname{Ext}^{1}(B / A, \operatorname{ker} p)=0$, Lemma 2.4 provides the lift!

Proposition 4.8. Suppose $\mathcal{F}$ is a locally cogenerated class which is closed under direct limits and contains a generator $G$. Furthermore, suppose $\mathcal{F}$ is closed under extensions and retracts. Then $\left(\mathcal{F}, \mathcal{F}^{\perp}\right)$ is a small cotorsion pair with $I$ as the set of generating monomorphisms.

Proof. First we show $\left(\mathcal{F}, \mathcal{F}^{\perp}\right)$ is a cotorsion pair cogenerated by the set $\operatorname{Gen}_{\kappa} \mathcal{F}$. So we show $\mathcal{F}={ }^{\perp}\left(\left(\mathbf{G e n}_{\kappa} \mathcal{F}\right)^{\perp}\right)$. First let $F \in \mathcal{F}$. By Lemma 4.3, $F$ is a transfinite extension of objects in $\mathbf{G e n}_{\kappa} \mathcal{F}$. It follows from the proof of Lemma 6.2 in [Hov02] that $\operatorname{Ext}(F, X)=0$ for any $X \in\left(\mathbf{G e n}_{\kappa} \mathcal{F}\right)^{\perp}$. So $F \in{ }^{\perp}\left(\left(\mathbf{G e n}_{\kappa} \mathcal{F}\right)^{\perp}\right)$. Conversely, say $X \in{ }^{\perp}\left(\left(\operatorname{Gen}_{\kappa} \mathcal{F}\right)^{\perp}\right)$. Factor $0 \rightarrow X$ using the small object argument (Theorem 2.1.14 of [Hov99]) as $0 \stackrel{i}{\rightarrow} F \stackrel{p}{\rightarrow} X$ where $i \in I$-cell and $p \in I$-inj. It follows from Remark 4.1 that $F \in \mathcal{F}$. It follows from Lemma 4.7 that $\operatorname{ker} p \in\left(\operatorname{Gen}_{\kappa} \mathcal{F}\right)^{\perp}$. Since $\operatorname{Ext}(X, \operatorname{ker} p)=0$ we see $p$ is a split epimorphism. Therefore $X$ is a retract of $F$. By the retract hypothesis $X \in \mathcal{F}$.

Now it follows from Lemma 4.7 and Lemma 3.4 that $\left(\mathcal{F}, \mathcal{F}^{\perp}\right)$ is a small cotorsion pair with generating monomorphisms the set $I$. 
4.2. Kaplansky classes. We now strengthen the idea of a locally cogenerated class to get the idea of a Kaplansky class. Our goal then is to show that a Kaplansky class in $\mathcal{G}$ gives rise to a model structure on $\mathrm{Ch}(\mathcal{G})$. The term "Kaplansky class" first appeared in [ELR02]. There it is defined for modules over a ring $R$ as a class $\mathcal{F}$ for which there is a regular cardinal $\kappa$ with the following property: Given $F \in \mathcal{F}$ and $x \in F$, there exists an $S \in \mathcal{F}$ with $x \in S \subseteq F$ and $|S| \leq \kappa$ and $F / S \in \mathcal{F}$. Below we formulate a version for abelian categories and then prove Theorem 4.12, the existence of an induced model category structure.

Definition 4.9. Let $\mathcal{F}$ be a class of objects in an abelian category and let $\kappa$ be a regular cardinal. We say $\mathcal{F}$ is a $\kappa$-Kaplansky class if the following property holds: Given $X \subseteq F \neq 0$ where $F \in \mathcal{F}$ and $X$ is $\kappa$-generated, there exists a $\kappa$-presentable object $S \neq 0$ such that $X \subseteq S \subseteq F$ and $S, F / S \in \mathcal{F}$. We say $\mathcal{F}$ is a Kaplansky class if it is a $\kappa$-Kaplansky class for some regular cardinal $\kappa$.

The requirement that we can find a $\kappa$-presentable object containing a $\kappa$-generated object in the definition of a $\kappa$-Kaplansky class may seem strange. However, in practice one usually needs to choose $\kappa$ to be very large in order to show that $\mathcal{F}$ is a $\kappa$-Kaplansky class. So why not just take $\kappa$ to be so large that the $\kappa$-generated and $\kappa$-presentable objects coincide? By Appendix B we can always find such a $\kappa$ as long as we are in a Grothendieck category. When this is the case, $\kappa$-generated becomes a good substitute for the notion of cardinality. For example, subobjects and quotient objects of $\kappa$-generated objects will again be $\kappa$-generated. (This follows from facts in Appendix A.) The method we just outlined is exactly what we will use in Section 6 to show that the class of flat quasi-coherent sheaves form a Kaplansky class. At any rate, we will need the definition of a $\kappa$-Kaplansky class to be as stated above in order to prove Proposition 4.11.

It is obvious that if $\mathcal{F}$ is a $\kappa$-Kaplansky class, then $\mathcal{F}$ is locally $\kappa$-cogenerated. We next prove that if $\mathcal{F}$ is a Kaplansky class, then the class of $\mathcal{F}$-complexes, denoted $\widetilde{\mathcal{F}}$, ought to be locally cogenerated as well.

Lemma 4.10. Let $\mathcal{G}$ be an abelian category and let $X$ be a chain complex in $\mathrm{Ch}(\mathcal{G})$. If $\kappa>\omega$ is a regular cardinal, then $X$ is $\kappa$-generated if and only if $X_{n}$ is $\kappa$-generated for each $n . X$ is $\omega$-generated (finitely generated) if and only if $X$ is bounded, above and below, and each $X_{n}$ is $\omega$-generated (finitely generated).

Proof. Say $\kappa$ is any regular cardinal, and let $n$ be an arbitrary integer. Let $X_{n}=$ $\sum_{i \in I} X_{i}$ where $X_{i} \subseteq X_{n}$. By Fact A.5 we wish to show $X_{n}=\sum_{i \in J} X_{i}$ where $J \subseteq I$ and $|J|<\kappa$. Each $X_{i}$ gives rise to a subcomplex $C\left(X_{i}\right)$ of $X$ by defining

$$
C\left(X_{i}\right)=\cdots \rightarrow d_{n+2}^{-1}\left(d_{n+1}^{-1}\left(X_{i}\right)\right) \rightarrow d_{n+1}^{-1}\left(X_{i}\right) \rightarrow X_{i} \rightarrow X_{n-1} \rightarrow X_{n-2} \rightarrow \cdots
$$

and furthermore $X=\sum_{i \in I} C\left(X_{i}\right)$. Since $X$ is $\kappa$-generated, Fact A.5 tells us $X=\sum_{i \in J} C\left(X_{i}\right)$ where $J \subseteq I$ and $|J|<\kappa$. Thus $X_{n}=\sum_{i \in J} X_{i}$. This shows that if $X$ is $\kappa$-generated, then $X_{n}$ is $\kappa$-generated in $\mathcal{G}$. For the special case of when $\kappa=\omega$, we still need to argue that $X$ is bounded. For this, write $X=\sum_{n=\mathbb{Z}} S_{n}$ where

$$
S_{n}=\cdots \rightarrow 0 \rightarrow X_{n} \rightarrow X_{n-1} \rightarrow X_{n-2} \rightarrow \cdots \rightarrow X_{-n} \rightarrow B_{-n-1} \rightarrow 0 \rightarrow \cdots
$$

Now Fact A.5 tells us $X=S_{n}$ for some $n$. So $X$ is bounded.

For the converse assume that each $X_{n}$ is $\kappa$-generated where $\kappa>\omega$. Let $X=$ $\sum_{i \in I} S_{i}$ where each $S_{i}$ is a subcomplex of $X$. Then $X_{n}=\sum_{i \in I}\left(S_{i}\right)_{n}$ and so there 
exists a set $J_{n} \subseteq I$ such that $\left|J_{n}\right|<\kappa$ and $X_{n}=\sum_{i \in J_{n}}\left(S_{i}\right)_{n}$. Let $J=\bigcup_{n \in \mathbb{Z}} J_{n}$. Then $X=\sum_{i \in J} S_{i}$ and $|J|<\kappa$ as desired. For the case of when $\kappa=\omega$ it is clear that we need the complex $X$ to be bounded so that the collection $J$ is finite.

Proposition 4.11. Let $\mathcal{F}$ be a $\kappa$-Kaplansky class. If $\mathcal{G}$ is locally $\kappa$-generated, then the class $\widetilde{\mathcal{F}}$ in $\mathrm{Ch}(\mathcal{G})$ is locally $\kappa$-cogenerated, as long as $\kappa>\omega$. (If $\kappa=\omega$, then $\widetilde{\mathcal{F}}$ is still $\kappa^{\prime}$-cogenerated for any regular $\kappa^{\prime}>\omega$.)

Proof. $\widetilde{\mathcal{F}}$ is the class of all exact chain complexes $F$ with $Z_{n} F \in \mathcal{F}$ for all $n$. Suppose $0 \neq F \in \mathcal{F}$ is given. We wish to construct a nonzero exact complex $S \subseteq F$ in such a way that each $Z_{n} S, Z_{n} F / Z_{n} S \in \mathcal{F}$ and each $S_{n}$ is $\kappa$-generated. It follows that $\widetilde{\mathcal{F}}$ is locally $\kappa$-cogenerated if $\kappa>\omega$. If $\kappa=\omega$ then $S$ may not be locally $\omega$-presentable because it need not be bounded. However in this case we still have $S \kappa^{\prime}$-generated for any regular $\kappa^{\prime}>\kappa$. So $\widetilde{\mathcal{F}}$ is locally $\kappa^{\prime}$-cogenerated.

Since $F \neq 0$ there must be some integer $n$ for which $Z_{n} F \neq 0$. We start by finding $0 \neq S_{n}^{\prime} \subseteq Z_{n} F$ with $S_{n}^{\prime} \kappa$-presentable and $S_{n}^{\prime} \in \mathcal{F}, Z_{n} F / S_{n}^{\prime} \in \mathcal{F}$. We set $S_{i}=0$ for all $i<n$ and set $S_{n}=S_{n}^{\prime}$. We now want to inductively build $S_{i}$ for $i>n$.

Since $\mathcal{G}$ is locally $\kappa$-generated, we can use Lemma 4.4 to find a $\kappa$-generated subobject $X_{n+1} \subseteq F_{n+1}$ for which $d_{\mid X_{n+1}}: X_{n+1} \rightarrow S_{n}^{\prime}$ is an epimorphism. Since $S_{n}^{\prime}$ is $\kappa$-presentable and $X_{n+1}$ is $\kappa$-generated and $\mathcal{G}$ is locally $\kappa$-generated, it follows from Lemma A.12 that ker $d_{\mid X_{n+1}}$ is $\kappa$-generated as well. Now using the definition of Kaplansky class, we can find a $\kappa$-presentable $S_{n+1}^{\prime}$ such that ker $d_{\mid X_{n+1}} \subseteq S_{n+1}^{\prime} \subseteq$ $Z_{n+1} F$ and $S_{n+1}^{\prime}, Z_{n+1} F / S_{n+1}^{\prime} \in \mathcal{F}$. Now

$$
d_{\mid X_{n+1}+S_{n+1}^{\prime}}: X_{n+1}+S_{n+1}^{\prime} \rightarrow S_{n}^{\prime}
$$

is an epimorphism whose kernel is $S_{n+1}^{\prime}$. So we set $S_{n+1}=X_{n+1}+S_{n+1}^{\prime}$. In this way we continue inductively to construct an exact subcomplex $0 \neq S \subseteq F$ with $Z_{n} S, Z_{n} F / Z_{n} S \in \mathcal{F}$ and $Z_{n} S \kappa$-presentable (and therefore $S_{n}$ is $\kappa$-generated by Facts A.3 and A.4).

Theorem 4.12. Let $\mathcal{G}$ be a locally $\kappa$-presentable Grothendieck category. Suppose $\mathcal{F}$ is a class of objects which satisfies the following:

(1) $\mathcal{F}$ is a $\kappa$-Kaplansky class.

(2) $\mathcal{F}$ contains a $\kappa$-presentable generator $G$ for $\mathcal{G}$.

(3) $\mathcal{F}$ is closed under direct limits, extensions and retracts.

(4) $d g \widetilde{\mathcal{F}} \cap \mathcal{E}=\widetilde{\mathcal{F}}$.

Then we have an induced model category structure on $\mathrm{Ch}(\mathcal{G})$ where the weak equivalences are the homology isomorphisms. The cofibrations (resp. trivial cofibrations) are the monomorphisms whose cokernels are in $d g \widetilde{\mathcal{F}}$ (resp. $\widetilde{\mathcal{F}}$ ). The fibrations (resp. trivial fibrations) are the epimorphisms whose kernels are in $d g \widetilde{\mathcal{C}}$ (resp. $\widetilde{\mathcal{C}}$ ), where $\mathcal{C}=\mathcal{F}^{\perp}$. Furthermore this model structure is cofibrantly generated. The generating cofibrations form the set

$$
I=\left\{0 \rightarrow D^{n}(G)\right\} \cup\left\{S^{n-1}(G) \rightarrow D^{n}(G)\right\} \cup\left\{S^{n}(A) \rightarrow S^{n}(B)\right\}
$$

where $A \rightarrow B$ ranges over all possible monomorphisms with $B$ a $\kappa$-generated object for which $B / A \in \mathcal{F}$. The generating trivial cofibrations are

$$
J=\left\{0 \rightarrow D^{n}(G)\right\} \cup\{X \rightarrow Y\}
$$


where $X \rightarrow \underset{\widetilde{\mathcal{F}}}{Y}$ ranges over all monomorphisms where $Y$ is a $\kappa$-generated complex and $Y / X \in \widetilde{\mathcal{F}}$

Proof. All of the work has been done. Since $\mathcal{F}$ is a locally cogenerated class, closed under direct limits, extensions and retracts, and containing a generator for $\mathcal{G}$, Proposition 4.8 tells us $(\mathcal{F}, \mathcal{C})$ is a small cotorsion pair. Now by Corollary 3.8 of [Gil04] we have the induced cotorsion pairs $(\widetilde{\mathcal{F}}, d g \widetilde{\mathcal{C}})$ and $(d g \widetilde{\mathcal{F}}, \widetilde{\mathcal{C}})$ of chain complexes. These cotorsion pairs are compatible by Corollary 3.9 above.

Having the cotorsion pairs of complexes $(d g \widetilde{\mathcal{F}} \cap \mathcal{E}, d g \widetilde{\mathcal{C}})$ and $(d g \widetilde{\mathcal{F}}, d g \widetilde{\mathcal{C}} \cap \mathcal{E})$ at hand we are in position to use Hovey's Theorem 2.2 from [Hov02]. By Proposition 3.8 we know that $(d g \widetilde{\mathcal{F}}, d g \widetilde{\mathcal{C}} \cap \mathcal{E})$ is small, so this cotorsion pair is complete by Corollary 6.6 of [Hov02]. Next $\widetilde{\mathcal{F}}=d g \widetilde{\mathcal{F}} \cap \mathcal{E}$ is locally cogenerated by Proposition 4.11. Furthermore, this class is closed under direct limits. To see this just apply the fact that direct limits are exact in $\mathcal{G}$, and $\mathcal{F}$ itself is closed under direct limits. It now follows from Propositions 4.8 that $(d g \widetilde{\mathcal{F}} \cap \mathcal{E}, \widetilde{\mathcal{C}})$ is small too and hence complete. Theorem 2.2 and Lemma 6.7 of [Hov02] tell us we have an induced model structure on $\mathrm{Ch}(\mathcal{G})$ which is cofibrantly generated. One can see that the weak equivalences, fibrations and cofibrations are as stated in the theorem by looking at Section 5 of [Hov02]. One can also see that the sets $I$ and $J$ are generating cofibrations and generating trivial cofibrations, respectively, by looking at Section 6 of [Hov02].

\section{Monoidal Model Structures}

Suppose our ground category $\mathcal{G}$ has a tensor product making it a closed symmetric monoidal Grothendieck category. We would like to have a condition on our Kaplansky class $\mathcal{F}$ which will guarantee that the model structure induced from Theorem 4.12 will be monoidal. We refer the reader to Chapter 4 of [Hov99] for a detailed discussion of monoidal model structures. There is also a nice discussion of the essential ideas in Section 7 of [Hov02]. In fact our proof below will rely on basic results from Section 7 of [Hov02]. Also, the crucial argument of the proof below, part (ii), is due to Hovey.

Theorem 5.1. Suppose $\mathcal{G}$ is a Grothendieck category with a closed symmetric monoidal structure $-\otimes_{\mathcal{G}}-$ and let $\mathcal{F}$ be a class of objects such that $\mathcal{G}$ and $\mathcal{F}$ satisfy the hypotheses of Theorem 4.12. Then the induced model structure on $\mathrm{Ch}(\mathcal{G})$ is monoidal with respect to the usual tensor product of chain complexes if the following conditions hold:

(1) Each object in $\mathcal{F}$ is flat. I.e., $F \otimes_{\mathcal{G}}-$ is exact for each $F \in \mathcal{F}$.

(2) If $F$ and $G$ both belong to $\mathcal{F}$, then $F \otimes_{\mathcal{G}} G$ also belongs to $\mathcal{F}$.

(3) $U \in \mathcal{F}$, where $U$ is the unit for the monoidal structure on $\mathcal{G}$.

Proof. Suppose the three stated conditions hold. We wish to check the four conditions of Theorem 7.2 from [Hov02]. In this case the four conditions translate to the following:

(i) Every cofibration is a pure injection in each degree.

(ii) If $X$ and $Y$ are in $d g \widetilde{\mathcal{F}}$, then $X \otimes Y$ is in $d g \widetilde{\mathcal{F}}$.

(iii) If $X$ is in $\widetilde{\mathcal{F}}$ and $Y$ is in $d g \widetilde{\mathcal{F}}$, then $X \otimes Y$ is in $\widetilde{\mathcal{F}}$.

(iv) The unit for the monoidal structure on $\operatorname{Ch}(\mathcal{G})$ is in $d g \widetilde{\mathcal{F}}$. 
Before we check each condition we make a few observations. First, as we can see from Proposition 3.8 , the hypotheses guarantee that $(d g \widetilde{\mathcal{F}}, \widetilde{\mathcal{C}})$ is a small cotorsion pair with cogenerating set consisting of spheres on objects from $\mathcal{F}$. That is, the cogenerating set consists of complexes of the form $S^{n}(F)$ where $F \in \mathcal{F}$. For the rest of the proof we will call any such object $S^{n}(F)$ an "F्F-sphere". Second, $d g \widetilde{\mathcal{F}}$ contains a set of generators for $\mathrm{Ch}(\mathcal{G})$ since if $G \in \mathcal{F}$ generates $\mathcal{G}$, then the set of complexes $D^{n}(G)$ generates $\operatorname{Ch}(\mathcal{G})$. (Each $D^{n}(F)$ is in $d g \widetilde{\mathcal{F}}$ by Lemma 3.4 of [Gil04].) It now follows from Lemma 3.6 that each complex in $d g \widetilde{\mathcal{F}}$ is a direct summand of a transfinite extension of $\mathcal{F}$-spheres. Lastly, we note that if $X \in d g \widetilde{\mathcal{F}}$, then $X \otimes-$ is exact. Indeed one can easily check that a complex $X$ is tensor exact if and only if $X_{n}$ is flat for each $n$. We now check the four conditions above.

(i) This follows from a basic property of flat objects. Cofibrations are monomorphisms with cokernels in $d g \widetilde{\mathcal{F}}$. In particular, the cokernel is flat in each degree. Therefore each cofibration is a pure injection in each degree. (See for example, the proof of Lemma XVI.3.1 in [Lan97].)

(ii) Step 1: We first show that if $X$ is a transfinite extension of $\mathcal{F}$-spheres and if $S^{n}(F)$ is an $\mathcal{F}$-sphere, then $X \otimes_{\mathrm{Ch}(\mathcal{G})} S^{n}(F)$ is in $d g \widetilde{\mathcal{F}}$. To see this first notice that the tensor product of two $\mathcal{F}$-spheres is again an $\mathcal{F}$-sphere and in particular is in $d g \widetilde{\mathcal{F}}$. Now say $X$ is a transfinite extension of a sequence such as

$$
X_{0} \hookrightarrow X_{1} \hookrightarrow \cdots \hookrightarrow X_{\alpha} \hookrightarrow X_{\alpha+1} \hookrightarrow \cdots
$$

where $X_{0}$ and each $X_{\alpha+1} / X_{\alpha}$ are $\mathcal{F}$-spheres. Since the functor $-\otimes_{\mathrm{Ch}(\mathcal{G})} S^{n}(F)$ is exact and also preserves direct limits, applying it to the above sequence will display $X \otimes_{\mathrm{Ch}(\mathcal{G})} S^{n}(F)$ as a transfinite extension of objects in $d g \widetilde{\mathcal{F}}$. Therefore it too is in $d g \widetilde{\mathcal{F}}$ by Lemma 6.2 of [Hov02].

Step 2: Now we see that if $X \in d g \widetilde{\mathcal{F}}$ and if $S^{n}(F)$ is an $\mathcal{F}$-sphere, then $X \otimes_{\mathrm{Ch}(\mathcal{G})}$ $S^{n}(F)$ is in $d g \widetilde{\mathcal{F}}$. The reason is that such an $X$ is a direct summand of a transfinite extension of $\mathcal{F}$-spheres. Since the functor $-\otimes_{\mathrm{Ch}(\mathcal{G})} S^{n}(F)$ commutes with direct sums, the result follows from the fact that $d g \widetilde{\mathcal{F}}$ is closed under direct summands. (The left side of any cotorsion pair is closed under direct summands.)

Step 3: Now we argue the same way to see that $X \otimes_{\mathrm{Ch}(\mathcal{G})} Y$ is in $d g \widetilde{\mathcal{F}}$ whenever $X$ and $Y$ are in $d g \widetilde{\mathcal{F}}$. For example if $Y$ is in $d g \widetilde{\mathcal{F}}$ then it is a retract of a transfinite extension of $\mathcal{F}$-spheres. As above, since $X \otimes_{\mathrm{Ch}(\mathcal{G})}-$ commutes with direct sums and $d g \widetilde{\mathcal{F}}$ is closed under taking direct summands we just need to show that $X \otimes_{\mathrm{Ch}(\mathcal{G})} Y$ is in $d g \widetilde{\mathcal{F}}$ when $X$ is in $d g \widetilde{\mathcal{F}}$ and $Y$ is a transfinite extension of $\mathcal{F}$-spheres. But in this case $Y$ can be viewed as the transfinite extension of a sequence such as

$$
Y_{0} \hookrightarrow Y_{1} \hookrightarrow \cdots \hookrightarrow Y_{\alpha} \hookrightarrow Y_{\alpha+1} \hookrightarrow \cdots
$$

where $Y_{0}$ and each $Y_{\alpha+1} / Y_{\alpha}$ are $\mathcal{F}$-spheres. Then we apply the functor $X \otimes_{\mathrm{Ch}(\mathcal{G})}-$ and use the fact from Step 2 to argue that $X \otimes_{\mathrm{Ch}(\mathcal{G})} Y$ is a transfinite extension of complexes in $d g \widetilde{\mathcal{F}}$. So $X \otimes_{\mathrm{Ch}(\mathcal{G})} Y$ is in $d g \widetilde{\mathcal{F}}$ too.

(iii) The approach is similar to our proof of (ii). Note that if $\mathcal{E}$ is the class of exact complexes in $\mathrm{Ch}(\mathcal{G})$, then $\mathcal{E}$ is closed under transfinite extensions and direct summands. One reason this is true is that $\mathcal{E}$ is the left side of the cotorsion pair $(\mathcal{E}, d g \widetilde{\mathcal{I}})$ where $d g \widetilde{\mathcal{I}}$ is the class of dg-injective complexes. 
Step 1: First we will prove that if $E$ is an exact complex in $\operatorname{Ch}(\mathcal{G})$ and $Y \in d g \widetilde{\mathcal{F}}$, then $E \otimes Y$ is exact. We leave it to the reader to see that, as in our proof of (ii), we can assume that $Y$ is a transfinite extension of $\mathcal{F}$-spheres. So suppose $Y$ is the direct limit of a sequence such as

$$
Y_{0} \hookrightarrow Y_{1} \hookrightarrow \cdots \hookrightarrow Y_{\alpha} \hookrightarrow Y_{\alpha+1} \hookrightarrow \cdots
$$

where $Y_{0}$ and each $Y_{\alpha+1} / Y_{\alpha}$ are $\mathcal{F}$-spheres. Since each short exact sequence

$$
0 \rightarrow Y_{\alpha} \rightarrow Y_{\alpha+1} \rightarrow Y_{\alpha+1} / Y_{\alpha} \rightarrow 0
$$

is pure in each degree, the sequence below is also exact:

$$
0 \rightarrow E \otimes Y_{\alpha} \rightarrow E \otimes Y_{\alpha+1} \rightarrow E \otimes Y_{\alpha+1} / Y_{\alpha} \rightarrow 0
$$

Now by applying the functor $E \otimes-$ to the entire sequence

$$
Y_{0} \hookrightarrow Y_{1} \hookrightarrow \cdots \hookrightarrow Y_{\alpha} \hookrightarrow Y_{\alpha+1} \hookrightarrow \cdots
$$

we can argue that $E \otimes Y$ is a transfinite extension of exact complexes and so is itself exact.

Step 2: Now say $X \in \widetilde{\mathcal{F}}$ and $Y \in d g \widetilde{\mathcal{F}}$. Then $X \in d g \widetilde{\mathcal{F}}$ by our assumption that $\widetilde{\mathcal{F}}=d g \widetilde{\mathcal{F}} \cap \mathcal{E}$. So by part (ii) we have that $X \otimes Y$ is in $d g \widetilde{\mathcal{F}}$. But by Step 1 of this proof we also have that $X \otimes Y$ is in $\mathcal{E}$. Therefore $X \otimes Y$ is in $\widetilde{\mathcal{F}}$.

(iv) Since $U \in \mathcal{F}$, we have from Lemma 3.4 of [Gil04] that $S(U)=S^{0}(U)$ is in the class $d g \widetilde{\mathcal{F}}$. Since $S(U)$ is the the unit for the tensor product on $\operatorname{Ch}(\mathcal{G})$ we are done.

\section{Chain COMPlexes of QuASI-COHERENT SheAves}

For the rest of the paper we turn to applications of Theorem 4.12 and Theorem 5.1. In this section we prove the existence of the flat model structure on $\mathrm{Ch}(\mathrm{Q} \operatorname{co}(X))$ when $X$ is a quasi-compact and semi-separated scheme.

So let $\left(X, \mathcal{O}_{X}\right)$ be a scheme on a topological space $X$. We denote the category of all sheaves of $\mathcal{O}_{X}$-modules by $\mathcal{O}_{X}$-Mod. We denote by $\mathrm{Qco}(X)$, the full subcategory of $\mathcal{O}_{X}$-Mod consisting of all quasi-coherent $\mathcal{O}_{X}$-modules. The class $\mathcal{F}$ of all flat quasi-coherent sheaves will be the Kaplansky class inducing the model structure. As far as the author can tell we need some assumptions on the scheme $X$ in order to satisfy the hypotheses of Theorem 4.12. From a study of the current literature it seems as though assuming $X$ is quasi-compact and semi-separated is the best we can do. In this case we see from [AJL97] or [Mur] that every quasi-coherent $\mathcal{O}_{X^{-}}$ module is the quotient of a flat quasi-coherent $\mathcal{O}_{X}$-module. We start this section by recalling some facts about the category $\mathrm{Qco}(X)$ as well as defining semi-separated. The author has used [Har77], [Lit82] and Appendix B of [TT90] as basic references on this material. We will also use recent results from [EE05].

The inclusion functor $\mathrm{Qco}(X) \rightarrow \mathcal{O}_{X}$-Mod is exact and $\mathrm{Qco}(X)$ is an abelian subcategory of $\mathcal{O}_{X}$-Mod. Therefore finite limits and colimits in $\mathrm{Qco}(X)$ are taken as in $\mathcal{O}_{X}$-Mod. As stated in the beginning of Appendix B of [TT90], $\mathrm{Qco}(X)$ is cocomplete with all small colimits taken as in $\mathcal{O}_{X}$-Mod. Therefore direct limits are exact in $\mathrm{Qco}(X)$. In fact $\mathrm{Q} \operatorname{co}(X)$ also has a set of generators making it a Grothendieck category. This last fact was stated as Lemma 2.1.7 in [Con00] and apparently is due to Gabber. A proof is not given in [Con00] but an independent proof can be found in [EE05]. 
Since we know $\mathrm{Qco}(X)$ has a generator and the inclusion functor $\mathrm{Qco}(X) \rightarrow$ $\mathcal{O}_{X}$-Mod preserves small colimits the inclusion has a right adjoint $Q: \mathcal{O}_{X}$-Mod $\rightarrow \mathrm{Qco}(X)$ by the special adjoint functor theorem. Thus $\mathrm{Q} \operatorname{co}(X)$ is a coreflective subcategory of $\mathcal{O}_{X}$-Mod. In particular, $Q$ satisfies a universal property dual in nature to the sheafification of a presheaf. One can now easily check that all small limits exist in $\mathrm{Qco}(X)$ and are taken by applying $Q$ to the usual limit taken in $\mathcal{O}_{X^{-}}$ Mod. Lastly, we recall that $\mathrm{Q} \operatorname{co}(X)$ is closed under extensions and tensor products taken in $\mathcal{O}_{X}$-Mod. The tensor product makes $\mathrm{Qco}(X)$ into a closed symmetric monoidal category. The closed structure is given by applying the functor $Q$ after the usual "sheafhom" functor.

A quasi-coherent $\mathcal{O}_{X}$-module $F$ is flat if the tensor functor $F \otimes_{\mathcal{O}_{X}}$ - is exact. Clearly the class of flat modules in $\mathrm{Qco}(X)$ is merely the class of flat modules in $\mathcal{O}_{X}$-Mod intersected with the class of all quasi-coherent $\mathcal{O}_{X}$-modules. We denote the class of all flat quasi-coherent $\mathcal{O}_{X}$-modules by $\mathcal{F}$. We point out that since flatness is defined in terms of the tensor product which is a left adjoint, $\mathcal{F}$ is closed under direct limits and retracts (summands). Furthermore, if

$$
0 \rightarrow F^{\prime} \rightarrow F \rightarrow F^{\prime \prime} \rightarrow 0
$$

is a short exact sequence of quasi-coherent sheaves with $F^{\prime \prime} \in \mathcal{F}$, then $F^{\prime} \in \mathcal{F}$ if and only if $F \in \mathcal{F}$. These assertions are all true by using categorical arguments with the tensor product, similar to those found in [Lan97] for modules over a ring. Alternatively, they can be shown using the characterization of flatness as a "stalkwise" property along with the fact that the corresponding property holds for modules over a ring. In any case, we point out that $\mathcal{F}$ is closed under retracts and transfinite extensions and also that $\mathcal{F}$ is resolving, meaning it is closed under taking kernels of epimorphisms between objects in $\mathcal{F}$.

We say a scheme $X$ is semi-separated if there exists an affine basis $\mathcal{V}=\left\{V_{\alpha}\right\}$ for $\operatorname{sp}(X)$ which is closed under finite intersections. The basis $\mathcal{V}$ is called a semiseparating affine basis of $X$. As pointed out in B.7 of [TT90] a semi-separated scheme is quasi-separated. Furthermore, a separated scheme is semi-separated with the set of all affine subsets serving as a semi-separating affine basis.

It is well-known that both $\mathcal{O}_{X}-\operatorname{Mod}$ and $\mathrm{Qco}(X)$ do not, in general, have enough projectives. While the category $\mathcal{O}_{X}$-Mod has a set of flat generators, the author does not know whether or not for a general scheme $X, \mathrm{Qco}(X)$ has a set of flat generators, or equivalently whether each quasi-coherent sheaf can be written as the quotient of a flat quasi-coherent sheaf. However, Proposition 1.1 of [AJL97] implies that this is indeed the case if we assume $X$ is a quasi-compact, separated scheme. In fact as pointed out by Daniel Murfet their proof works for any quasi-compact semi-separated scheme $X$. Murfet's proof can be found as Proposition 16 in [Mur]. Since the quasi-compact semi-separated hypothesis is the best one the author has found for the existence of enough flats in $\mathrm{Qco}(X)$ we will build the flat model structure on $\mathrm{Ch}(\mathrm{Qco}(X))$ under these assumptions on $X$.

The following subsections will contain lemmas breaking down the proof of Theorem 6.7 which of course amounts to checking the four hypotheses of Theorem 4.12 for the class $\mathcal{F}$ of flat modules in $\mathrm{Q} \operatorname{co}(X)$. We start with the Kaplansky class condition. As we will see, it readily follows from recent work of Enochs and Estrada that $\mathcal{F}$ is indeed a Kaplansky class for any scheme $X$ [EE05]. Since the term Kaplansky class was not used in [EE05] we now check carefully that their work proves $\mathcal{F}$ is a Kaplansky class in the sense of Definition 4.9. 
6.1. Quasi-coherent modules on a representation of a quiver. By a quiver we mean a directed graph $Q=(V, E)$ where $V$ is the set of vertices and $E$ is the set of edges. By a representation of $Q$, we will mean a functor $R: Q \rightarrow$ CRng where $Q$ is thought of as a category in the obvious way and CRng is the category of commutative rings with identity. Let $R$ be a representation of $Q$. By an $R$ module $M$ we will mean an $R(v)$-module, $M(v)$, for each $v \in V$, and an $R(v)$-linear map $M(e): M(v) \rightarrow M(w)$ for each edge $e: v \rightarrow w$ in $E$. We refer the reader to Section 2 of [EE05] for the definition of a flat representation and the definition of a quasi-coherent $R$-module. It is also pointed out there that the category $\mathcal{C}$ of quasi-coherent $R$-modules is a Grothendieck category when $R$ is flat.

We define the cardinality of an $R$-module as

$$
|M|=\left|\coprod_{v \in V} M(v)\right|
$$

Lemma 6.1. Let $R$ be a flat representation of a quiver $Q=(V, E)$ and $M$ be a quasi-coherent $R$-module. Let $\kappa$ be a regular cardinal for which $\kappa>|R(v)|$ for all $v$ and $\kappa>\max \{|E|,|V|\}$. Then the following are equivalent.

(1) $|M|<\kappa$.

(2) $M$ is $\kappa$-generated.

(3) $M$ is $\kappa$-presentable.

Proof. $(1) \Rightarrow(2)$. We use Fact A.5. Suppose $|M|<\kappa$ and say $M=\sum_{i \in I} M_{i}$ is a $\kappa$-filtered union of quasi-coherent submodules. For each $x \in M$, there corresponds some $i \in I$ such that $x \in M_{i}$. But $\sum_{i \in I} M_{i}$ is $\kappa$-filtered and $|M|<\kappa$, so there exists $i \in I$ such that $M=M_{i}$.

$(2) \Rightarrow(1)$. Let $\mathcal{S}$ be the collection of all subsets $S \subseteq \coprod_{v \in V} M(v)$ such that $|S|<\kappa$. For each $S \in \mathcal{S}$, let $M_{S}$ represent the quasi-coherent submodule generated by $S$. Then $\left|M_{S}\right|<\kappa$. (One way to see this is to use Proposition 3.3 of [EE05]. All $\leq$ signs in that Proposition can be changed to $<$ signs.) Note that $(\mathcal{S}, \subseteq)$ is $\kappa$-filtered and in fact $M$ is the $\kappa$-filtered union $\sum_{S \in \mathcal{S}} M_{S}$. By Fact A.5, $M=M_{S}$ for some $S \in \mathcal{S}$.

$(3) \Rightarrow(2)$ is automatic. We now prove $(2) \Rightarrow(3)$, using that (1) iff (2). First we point out that the category of quasi-coherent $R$-modules is locally $\kappa$-generated. Indeed each $M$ can be expressed as the $\kappa$-filtered union $M=\sum_{S \in \mathcal{S}} M_{S}$ where each $M_{S}$ is $\kappa$-generated as in the last paragraph. Therefore, we may use the characterization of $\kappa$-presentable objects in Fact A.12. Suppose $M$ is $\kappa$-generated and $N \rightarrow M$ is an epimorphism with $N$ a $\kappa$-generated $R$-module. Then $|N|<\kappa$. So of course $|\operatorname{ker}(N \rightarrow M)|<\kappa$ (kernels are computed componentwise), which means $\operatorname{ker}(N \rightarrow M)$ is $\kappa$-generated. This proves $M$ is $\kappa$-presentable.

An $R$-module $M$ is called flat if each $M(v)$ is a flat $R(v)$-module.

Lemma 6.2. Let $R$ be a flat representation of a quiver $Q=(V, E)$. Let $\kappa$ be a regular cardinal for which $\kappa>|R(v)|$ for all $v$ and $\kappa>\max \{|E|,|V|\}$. Then the class of all flat quasi-coherent $R$-modules constitute a $\kappa$-Kaplansky class.

Proof. This follows immediately from Lemma 6.1 along with the Proposition 3.3 of [EE05]. Again, all $\leq$ signs can be changed to $<$ signs in the statement and proof of Proposition 3.3 of [EE05]. 
6.2. A category equivalent to $\mathbf{Q} \operatorname{co}(\mathbf{X})$. If $X$ is a scheme, then the collection of all affine open subsets determines a directed graph, $\mathcal{A}_{X}$. The opposite graph (reverse all arrows) is also a quiver which we will denote $Q_{X}$. We get a (flat) representation $R$ by letting $R(U)=\mathcal{O}_{X}(U)$ for each $U \in \mathcal{A}_{X}$ and using the restriction maps to get $R(V) \rightarrow R(U)$ whenever $U \subseteq V$. In the same way, a quasi-coherent sheaf $S$ on $X$ gives rise to a quasi-coherent $R$-module $M$ by letting $M(U)=S(U)$ for $U \in \mathcal{A}_{X}$. In fact, as explained in [EE05], the category $\mathcal{C}$ of quasi-coherent $R$-modules is categorically equivalent to $\mathrm{Qco}(X)$. Furthermore, the equivalence restricts to an equivalence between the flat objects in $\mathcal{C}$ and the flat objects of $\mathrm{Q} \operatorname{co}(X)$.

Lemma 6.3. Let $\mathcal{A}_{0} \subseteq \mathcal{A}$ and $\mathcal{B}_{0} \subseteq \mathcal{B}$ where $\mathcal{A}$ and $\mathcal{B}$ are abelian categories. Let $G: \mathcal{A} \rightarrow \mathcal{B}$ be an additive functor which is an equivalence. If $G$ restricts to an equivalence between $\mathcal{A}_{0}$ and $\mathcal{B}_{0}$, then $\mathcal{A}_{0}$ is a $\kappa$-Kaplansky class if and only if $\mathcal{B}_{0}$ is a $\kappa$-Kaplansky class.

Proof. Since $G: \mathcal{A} \rightarrow \mathcal{B}$ is an equivalence, there exists $H: \mathcal{B} \rightarrow \mathcal{A}$ such that $H G \cong$ $1_{\mathcal{A}}$ and $G H \cong 1_{\mathcal{B}}$. Furthermore, $G$ is both a left and right adjoint of $H$ (and so each preserves colimits). We start by showing that $G$ preserves $\kappa$-generated and $\kappa$ presentable objects. Indeed if $X \in \mathcal{A}$ is $\kappa$-presentable then the functor $\mathcal{A}(X, H(-))$ preserves $\kappa$-filtered colimits. But $\mathcal{A}(X, H(-)) \cong \mathcal{B}(G(X),-)$, so $G(X)$ must be $\kappa$-presentable. On the other hand, if $G(X)$ is $\kappa$-presentable, then $\mathcal{B}(G(X), G(-))$ preserves $\kappa$-filtered colimits. Therefore, $\mathcal{B}(G(X), G(-)) \cong \mathcal{A}(H G(X),-)$ preserves $\kappa$-filtered colimits and $H G(X) \cong X$ must be $\kappa$-presentable. The same proof (but with $\kappa$-filtered colimits of monomorphisms) shows that an equivalence preserves $\kappa$-generated objects.

Now we suppose $G$ restricts to an equivalence between $\mathcal{A}_{0}$ and $\mathcal{B}_{0}$ and that $\mathcal{B}_{0}$ is a $\kappa$-Kaplansky class of $\mathcal{B}$. We will show that $\mathcal{A}_{0}$ is a $\kappa$-Kaplansky class of $\mathcal{A}$. So let $X \subseteq F \neq 0$ where $F \in \mathcal{A}_{0}$ and $X$ is a $\kappa$-generated object in $\mathcal{A}$. Then $G(X) \subseteq G(F) \neq 0$ and $G(X)$ is $\kappa$-generated and $G(F) \in \mathcal{B}_{0}$. Since $\mathcal{B}_{0}$ is a $\kappa$ Kaplansky class, there exists a nonzero $\kappa$-presentable object $S \in \mathcal{B}_{0}$ for which $G(X) \subseteq S \subseteq G(F)$ and $G(F) / S \in \mathcal{B}_{0}$. Therefore $H(S) \in \mathcal{A}_{0}$ is a nonzero $\kappa$ presentable object for which $X=H G(X) \subseteq H(S) \subseteq H G(F)=F$ and $F / H(S)=$ $H G(F) / H(S)=H(G F / S) \in \mathcal{A}_{0}$.

Proposition 6.4. Let $\left(X, \mathcal{O}_{X}\right)$ be quasi-compact and semi-separated scheme. Let $\mathcal{F}$ be the class of flat quasi-coherent $\mathcal{O}_{X}$-modules and define $\mathcal{C}=\mathcal{F}^{\perp}$. Then $\mathcal{F}$ is a Kaplansky class and $(\mathcal{F}, \mathcal{C})$ is a small cotorsion pair.

Proof. It follows from the lemmas above that $\mathcal{F}$ is a Kaplansky class. In particular $\mathcal{F}$ is locally cogenerated. Furthermore as explained in the introduction to this section, $\mathcal{F}$ is closed under transfinite extensions and retracts and contains a generator. Therefore $(\mathcal{F}, \mathcal{C})$ is a small cotorsion pair by Proposition 4.8.

6.3. Exact dg-flat complexes are flat. Let $\left(X, \mathcal{O}_{X}\right)$ be a quasi-compact semiseparated scheme. For what follows we fix a semi-separating affine basis $\mathcal{V}$ and again let $\mathcal{F}$ be the class of all flat modules in $\operatorname{Qco}(X)$. We will prove that $\widetilde{\mathcal{F}}=d g \widetilde{\mathcal{F}} \cap \mathcal{E}$ where $\mathcal{E}$ is the class of all exact chain complexes.

Recall that if $f: Y \rightarrow Z$ is any morphism of schemes then by Proposition II.5.8 of [Har77] the inverse image functor $f^{*}$ preserves quasi-coherence. In particular, if $V_{\alpha} \in \mathcal{V}$ and $j: V_{\alpha} \rightarrow X$ is the inclusion, then we have the functor $j^{*}: \operatorname{Qco}(X) \rightarrow$ 
$\mathrm{Q} \operatorname{co}\left(\left.X\right|_{V_{\alpha}}\right)$. In this case the functor is merely restriction of a quasi-coherent $\mathcal{O}_{X^{-}}$ module to the affine subset $V_{\alpha}$ and it is clear that $j^{*}$ is an exact functor.

On the other hand, recall that the direct image functor $f_{*}$ does not always preserve quasi-coherence. However it is easy to see that $j_{*}$ does preserve quasicoherence. Indeed $j$ is separated since it is an open immersion (see Theorem 1.17 of [Lit82]). Also since affine subsets are quasi-compact we see by Proposition 1.51 of [Lit82] (with our semi-separating basis $\mathcal{V}$ used as the affine open cover required in the Proposition), that $j_{*}$ preserves quasi-coherence.

Lemma 6.5. Let $\left(X, \mathcal{O}_{X}\right)$ be a semi-separated scheme with semi-separating affine basis $\mathcal{V}=\left\{V_{\alpha}\right\}$. Let $V_{\alpha} \in \mathcal{V}$ and $j: V_{\alpha} \rightarrow X$ be the inclusion. Then $j^{*}: \operatorname{Qco}(X) \rightarrow$ $\mathrm{Q} \operatorname{co}\left(\left.X\right|_{V_{\alpha}}\right)$ is left adjoint to $j_{*}: \mathrm{Qco}\left(\left.X\right|_{V_{\alpha}}\right) \rightarrow \mathrm{Qco}(X)$. Furthermore $j_{*}$ is exact and preserves cotorsion objects.

Proof. It is a standard fact that inverse image functors are left adjoint to direct image functors. For example, see Section II.5 of [Har77]. In particular, $j^{*}$ is left adjoint to $j_{*}$. Since $j_{*}$ is a right adjoint it is left exact. We will show that $j_{*}$ preserves surjections. So suppose we have a surjection $\mathcal{F} \rightarrow \mathcal{G}$ of quasi-coherent sheaves in $\mathrm{Q} \operatorname{co}\left(\left.X\right|_{V_{\alpha}}\right)$. Then given any affine subset $V_{\gamma} \subseteq V_{\alpha}$, we know $\mathcal{F}\left(V_{\gamma}\right) \rightarrow \mathcal{G}\left(V_{\gamma}\right)$ is a surjection. It follows that for any affine $V_{\beta} \in \mathcal{V},\left[j_{*}(\mathcal{F})\right]\left(V_{\beta}\right) \rightarrow\left[j_{*}(\mathcal{G})\right]\left(V_{\beta}\right)$ is surjective. Indeed the definition of $\left[j_{*}(\mathcal{F})\right]\left(V_{\beta}\right) \rightarrow\left[j_{*}(\mathcal{G})\right]\left(V_{\beta}\right)$ is just $\mathcal{F}\left(V_{\gamma}\right) \rightarrow \mathcal{G}\left(V_{\gamma}\right)$ where $V_{\gamma}=V_{\alpha} \cap V_{\beta} \in \mathcal{V}$. It follows that $j_{*}$ is exact since $\mathcal{V}$ is a basis for the topology on $X$.

We now show that for any quasi-coherent sheaf $\mathcal{F} \in \mathrm{Q} \operatorname{co}(X)$ and any quasicoherent sheaf $\mathcal{C} \in \mathrm{Qco}\left(\left.X\right|_{V_{\alpha}}\right)$ we have an isomorphism

$$
\operatorname{Ext}^{n}\left(j^{*} \mathcal{F}, \mathcal{C}\right) \cong \operatorname{Ext}^{n}\left(\mathcal{F}, j_{*} \mathcal{C}\right)
$$

First note that $j_{*}$ being the right adjoint of an exact functor preserves injective objects by Proposition 2.3.10 of [Wei94]. Since $j_{*}$ itself is also exact it preserves injective resolutions. The result now follows by taking an injective resolution of $\mathcal{C}$, applying $j_{*}$ to the resolution, and then applying the adjoint relationship between $j_{*}$ and $j^{*}$.

Now suppose $\mathcal{C} \in \operatorname{Qco}\left(\left.X\right|_{V_{\alpha}}\right)$ is cotorsion and $\mathcal{F}$ is a flat quasi-coherent $\mathcal{O}_{X^{-}}$ module. We wish to show that $j_{*} \mathcal{C}$ cotorsion. But $j^{*} \mathcal{F}$ is clearly flat since $j^{*}$ is just restriction, and so $\operatorname{Ext}^{1}\left(\mathcal{F}, j_{*} \mathcal{C}\right) \cong \operatorname{Ext}^{1}\left(j^{*} \mathcal{F}, \mathcal{C}\right)=0$. Therefore $j_{*} \mathcal{C}$ is cotorsion.

Proposition 6.6. Let $\left(X, \mathcal{O}_{X}\right)$ be a semi-separated scheme with semi-separating affine basis $\mathcal{V}=\left\{V_{\alpha}\right\}$ and let $\mathcal{F}$ be the class of flat quasi-coherent $\mathcal{O}_{X}$-modules. Then $\widetilde{\mathcal{F}}=d g \widetilde{\mathcal{F}} \cap \mathcal{E}$ where $\mathcal{E}$ is the class of exact complexes.

Proof. We saw in Proposition 6.4 that $(\mathcal{F}, \mathcal{C})$ is a cotorsion pair. Now Lemma 3.10 of [Gil04] says that $\widetilde{\mathcal{F}} \subseteq d g \widetilde{\mathcal{F}} \cap \mathcal{E}$. So we wish to prove $\widetilde{\mathcal{F}} \supseteq d g \widetilde{\mathcal{F}} \cap \mathcal{E}$. We let $Y$ be an exact dg-flat complex of quasi-coherent $\mathcal{O}_{X}$-modules. We want to show that it is a flat complex. I.e. that is has flat cycles in each degree. One way to do this is to show that the complex $j^{*}(Y)$ is a flat complex of $\left.\mathcal{O}\right|_{V_{\alpha}}$-modules for each inclusion $j: V_{\alpha} \rightarrow X$ where $V_{\alpha} \in \mathcal{V}$. So let $j: V_{\alpha} \rightarrow X$ be such an inclusion. Then by the usual equivalence between the category of $A_{\alpha}$-modules and the category of quasi-coherent sheaves on $\tilde{A_{\alpha}}$ where $A_{\alpha}$ is a ring in which $\left.\tilde{A_{\alpha}} \cong \mathcal{O}\right|_{V_{\alpha}}$, the chain complex $j^{*}(Y)=\left.Y\right|_{V_{\alpha}}$ corresponds to a chain complex of $A_{\alpha}$-modules. Since the equivalence preserves flat objects by Proposition III.9.2 of [Har77] it also preserves cotorsion objects. Therefore, the complex of $A_{\alpha}$-modules corresponding to $j^{*}(Y)$ is 
flat (respectively dg-flat) if and only if $j^{*}(Y)$ is flat (respectively dg-flat). Finally, since it is already known from [Gil04] that exact, dg-flat complexes of $A_{\alpha}$-modules are flat complexes we will be able to conclude that $j^{*}(Y)$ is flat by just proving it is exact and dg-flat.

It is clear that $j^{*}(Y)$ is exact so we will show that $j^{*}(Y)$ is a dg-flat complex. So let $j^{*}(Y) \rightarrow C$ be a morphism where $C$ is a cotorsion complex of quasi-coherent $\mathcal{O}_{V_{\alpha}}$-modules. (This means that $C$ is exact and each cycle is cotorsion.) Using the definition of dg-flat we wish show this map is null homotopic. But using the adjoint property of $j^{*}$ and $j_{*}$ gives us a morphism $Y \rightarrow j_{*}(C)$ of complexes. Since $j_{*}(-)$ is exact and preserves cotorsion modules by Lemma 6.5 we see that $j_{*}(C)$ is a cotorsion complex and by definition of dg-flat $Y \rightarrow j_{*}(C)$ is null homotopic. From this we can deduce that $j^{*}(Y) \rightarrow C$ is also null homotopic.

6.4. The main theorem. We are now ready to prove the main theorem. By applying Definition 3.3 of [Gil04] to the cotorsion pair $(\mathcal{F}, \mathcal{C})$ we get four classes of chain complexes in $\mathrm{Ch}(\mathrm{Q} \operatorname{co}(X))$. We call the complexes in $d g \widetilde{\mathcal{F}}$ the $d g$-flat complexes, and the complexes in $\widetilde{\mathcal{F}}$ the flat complexes. We call the complexes in $d g \widetilde{\mathcal{C}}$ the $d g$-cotorsion complexes, and the complexes in $\widetilde{\mathcal{C}}$ the cotorsion complexes.

Theorem 6.7. Let $X$ be a quasi-compact semi-separated scheme and let $\mathcal{F} \subseteq$ $\mathrm{Qco}(X)$ be the class of flat quasi-coherent sheaves. Then we have a cofibrantly generated model category structure on $\mathrm{Ch}(\mathrm{Qco}(X))$ which is described as follows: The weak equivalences are the homology isomorphisms. The cofibrations (resp. trivial cofibrations) are the monomorphisms whose cokernels are dg-flat complexes (resp. flat complexes). The fibrations (resp. trivial fibrations) are the epimorphisms whose kernels are dg-cotorsion complexes (resp. cotorsion complexes). Furthermore, this model structure is monoidal with respect to the usual tensor product of chain complexes.

Proof. From B.3 of [TT90] we see that if $X$ is quasi-compact and quasi-separated, then $\mathrm{Qco}(X)$ is locally finitely presentable [TT90]. That is, $\mathrm{Qco}(X)$ is locally $\kappa$ presentable (and hence $\kappa$-generated) for any regular cardinal $\kappa$. Since $\mathrm{Qco}(X)$ is a Grothendieck category it has a generator. By writing this generator as the quotient of a flat quasi-coherent $\mathcal{O}_{X}$-module we obtain a flat generator. By Lemmas 6.2 and 6.3 we can find arbitrarily large regular cardinals $\kappa$ for which $\mathcal{F}$ is a $\kappa$-Kaplansky class. In light of Fact A.2 we can find a single regular cardinal $\kappa$ such that (i) $\mathrm{Qco}(X)$ is locally $\kappa$-presentable, (ii) $\mathcal{F}$ is a $\kappa$-Kaplansky class, and (iii) $\mathcal{F}$ contains a $\kappa$-presentable generator. This verifies properties (1) and (2) of Theorem 4.12. Property (3) holds as explained in the introduction to this section. Property (4) is Proposition 6.6. This finishes the proof of the existence of the flat model structure on $\mathrm{Ch}(\mathrm{Qco}(X))$.

We now check that the model structure is monoidal. Condition (1) of Theorem 5.1 is trivial. Condition (2) is true since the tensor product of two flat quasicoherent sheaves is again a flat quasi-coherent sheaf. The unit for the tensor product is the structure sheaf $\mathcal{O}_{X}$. It is both quasi-coherent and flat and so condition (3) holds.

Many questions come to mind that the author has not been able to figure out or has not had time to consider. First, is there an easier proof that $\mathrm{Qco}(X)$ has enough flat objects when $X$ is quasi-compact and semi-separated? Can we construct 
some actual flat generators for $\mathrm{Qco}(X)$ that are strongly dualizable in $\mathcal{D}(\mathrm{Qco}(X))$ ? Is $\mathcal{D}(\mathrm{Q} c o(X))$ a stable homotopy category in the sense of [HPS97]? What are the implications of May's additivity theorem in [May3]? Can one easily treat the derived inverse and direct image functors from the model category viewpoint?

\section{Other APPLICATIONS}

In this section we point out other applications of Theorem 4.12. In particular we will show that all model structures the author has previously constructed in [Gil04] and [Gil06] as well as the canonical "injective" model structure can each be construed as corollaries to Theorem 4.12. Note that condition (1) (the Kaplansky class condition) in Theorem 4.12 is defined in terms of $\kappa$-generated and $\kappa$-presentable objects. In practice it may be cumbersome to work with the definitions of $\kappa$-generated or $\kappa$-presentable. In each corollary below we find it convenient to pick $\kappa$ large enough so that the $\kappa$-generated objects and $\kappa$-presentable objects coincide. In the case when $\mathcal{G}$ is a concrete category we go further and relate the notions of $\kappa$-generated and $\kappa$-presentable to the cardinality of the underlying set. As mentioned after the definition of Kaplansky class in Section 4.2, $\kappa$-generated and $\kappa$-presentable become good categorical replacements for the notion of cardinality.

Corollary 7.1. Let $\mathcal{G}$ be any Grothendieck category and let $\mathcal{A}$ be the class of all objects in $\mathcal{G}$. Then $\mathcal{A}$ is a Kaplansky class and it induces the injective model structure on $\mathrm{Ch}(\mathcal{G})$. Furthermore this model structure is cofibrantly generated.

Proof. $\mathcal{G}$ is locally $\kappa$-presentable for some regular cardinal $\kappa$ by Proposition 3.10 of [Bek00]. Note $\mathcal{A}^{\perp}=\mathcal{I}$ is the class of injectives and $(\mathcal{A}, \mathcal{I})$ is the "injective cotorsion pair". In order to use Theorem 4.12 we would like to say that $\mathcal{A}$ is a $\kappa$-Kaplansky class, but the author suspects that this is just not true. (If it were, then clearly every locally $\kappa$-generated Grothendieck category would be locally $\kappa$ presentable. See the paragraph before Fact A.7 in Appendix A.) In any case there is a trick: Pick $\kappa^{\prime}$ to be a regular cardinal large enough so that (i) $\mathcal{G}$ is locally $\kappa^{\prime}$-presentable, (ii) the class of $\kappa^{\prime}$-generated objects coincides with the class of $\kappa^{\prime}$ presentable objects, and (iii) there is a $\kappa^{\prime}$-presentable generator for $\mathcal{G}$. Condition (i) is possible by FactA.8, condition (ii) is possible by Appendix B, and condition (iii) is possible by Fact A.2. Now using $\kappa^{\prime}$ and $\mathcal{A}$ we can easily check the conditions of Theorem 4.12. Conditions (1) and (3) hold trivially and condition (2) holds by our choice of $\kappa^{\prime}$. Finally if $I \in \widetilde{\mathcal{I}}$, it is a well-known fact that every chain map into $I$ is null homotopic. Therefore $d g \widetilde{\mathcal{A}}$ is simply the class of all chain complexes in $\mathrm{Ch}(\mathcal{G})$. It is also clear that $\widetilde{\mathcal{A}}=\mathcal{E}$, so condition (4) from Theorem 4.12 holds too. The conclusion of Theorem 4.12 translates to the usual injective model structure where the cofibrations are the monomorphisms and the fibrations are the epimorphisms with dg-injective kernels.

It was in [ELR02] that the term "Kaplansky class" first appeared. The reasoning is that the class of projective modules (over a ring $R$ ) is a Kaplansky class by Theorem 1 of [Kap58]. There is of course a "projective" model structure on $\mathrm{Ch}(R)$ which is associated to this Kaplansky class. Unfortunately, we can not recapture this model structure using Theorem 4.12. The only problem is that the class of projectives is not closed under direct limits. This suggests that there may be a better theorem than 4.12 which excludes the direct limit hypothesis. The first idea would be to replace the "closed under direct limits" hypothesis with a "closed 
under transfinite extensions" hypothesis. However, how would we get a lemma similar to 4.3 ?

Another (monoidal) model structure on $\mathrm{Ch}(R)$ which can be obtained using Theorem 4.12 is the flat model structure which first appeared in [Gil04].

Corollary 7.2. Let $\mathcal{G}=R$-Mod where $R$ is a commutative ring with 1 and let $\mathcal{F}$ be the class of flat modules. Then $\mathcal{F}$ is a Kaplansky class and it induces a model structure on $\mathrm{Ch}(\mathcal{G})$ called the flat model structure. The cofibrations are the monomorphisms with dg-flat cokernels and the fibrations are the epimorphisms with $d g$-cotorsion kernels. Furthermore this model structure is cofibrantly generated and monoidal with respect to the usual tensor product of chain complexes.

Proof. First we note that $\mathcal{G}$ is locally finitely presentable. For a proof of this fact, see the footnote to Theorem 4.34 of [Lam99]. So $\mathcal{G}$ is locally $\kappa$-presentable for every regular cardinal $\kappa$ by Fact A.8. We let $\kappa$ be a regular cardinal with $\kappa>|R|$ and argue that $\mathcal{F}$ is a $\kappa$-Kaplansky class.

So let $X \subseteq F \neq 0$ where $X$ is $\kappa$-generated and $F$ is flat. By Lemma B.1, $|X|<\kappa$. For each $x \in X$ we use Lemma 2 of [BBE01] to find a flat submodule $F_{x} \subseteq F$ with $x \in F_{x},\left|F_{x}\right|<\kappa$ and $F / F_{x} \in \mathcal{F}$. The direct union $\bigcup_{x \in X} F_{x}$ contains $X$ and is flat. Furthermore

$$
F /\left(\bigcup_{x \in X} F_{x}\right) \cong \lim _{x \in X} F / F_{x}
$$

is flat. Finally, since $\left|\bigcup_{x \in X} F_{x}\right|<\kappa$, Lemmas B.1 and B.2 allow us to conclude that $\mathcal{F}$ is a $\kappa$-Kaplansky class.

This proves property (1) of Theorem 4.12 and property (2) is clearly true since $R$ is a flat $R$-module. Proposition XVI.3.1 of [Lan97] tells us that $\mathcal{F}$ is closed under retracts (direct summands). It is also a standard fact about flat modules that $\mathcal{F}$ is closed under extensions and direct limits. So it is left to check property (4) of Theorem 4.12. By Proposition XVI.3.4 of [Lan97] $\mathcal{F}$ is closed under taking kernels of epimorphisms. Referring to Definition 3.11 of [Gil04] this says that the cotorsion pair $(\mathcal{F}, \mathcal{C})$ (where $\mathcal{C}$ is the class of all cotorsion modules) is hereditary. Corollary 3.13 of [Gil04] now tells us that $\widetilde{\mathcal{F}}=d g \widetilde{\mathcal{F}} \cap \mathcal{E}$.

Thus we have a cofibrantly generated model structure on $\mathrm{Ch}(R)$ where the cofibrations are the monomorphisms with cokernels in $d g \widetilde{\mathcal{F}}$ (dg-flat complexes) and the fibrations are the epimorphisms with kernels in $d g \widetilde{\mathcal{C}}$ (dg-cotorsion complexes).

It is easy to see that the model structure is monoidal using Theorem 5.1. The tensor product of two flat modules is clearly flat and the unit $R$ is flat.

As described in [Gil06] the (monoidal) flat model structure of Corollary 7.2 generalizes to the category $\mathrm{Ch}\left(\mathcal{O}_{X}\right.$-Mod) of complexes of $\mathcal{O}_{X}$-modules where $\mathcal{O}_{X}$ is a ringed space. It too is obtained from a Kaplansky class as we will see next in Corollary 7.8. The first lemma below concerns cotorsion modules and skyscraper sheaves. We now recall the concept of a skyscraper sheaf.

Let $\mathcal{O}_{X}$ be a ringed space, $p \in X$ be a point, and $M$ be an $\mathcal{O}_{p}$-module. The skyscraper sheaf $S_{p}(M)$ is the sheaf on $X$ defined by $U \mapsto M$ if $p \in U$ and $U \mapsto 0$ if $p \notin U$. It is in fact an $\mathcal{O}_{X}$-module by viewing $M$ as an $\mathcal{O}(U)$-module via the ring homomorphism $\mathcal{O}(U) \rightarrow \mathcal{O}_{p}$. One can check that $\left[S_{p}(M)\right]_{q}=M$ for each $q \in \overline{\{p\}}$ and $\left[S_{p}(M)\right]_{q}=0$ for each $q \notin \overline{\{p\}}$. It is also standard that $S_{p}(-)$ is an exact functor from the category $\mathcal{O}_{p}$-Mod to the category $\mathcal{O}_{X}$-Mod and is right adjoint to 
the (also exact) "stalk functor" which sends a $\mathcal{O}_{X}$-module $F$ to the $\mathcal{O}_{p}$-module $F_{p}$. Therefore $S_{p}(-)$ preserves injective objects by Proposition 2.3.10 of [Wei94].

Lemma 7.3. Let $\mathcal{O}_{X}$ be a ringed space and $p \in X$ be a point. The skyscraper functor $S_{p}(-)$ preserves cotorsion objects.

Proof. First notice that for any $\mathcal{O}_{X}$-module $F$ and any $\mathcal{O}_{p}$-module $C$ we have an isomorphism $\operatorname{Ext}^{n}\left(F_{p}, C\right) \cong \operatorname{Ext}^{n}\left(F, S_{p}(C)\right)$. (This can be proved using the adjoint relationship discussed above along with the fact that $S_{p}(-)$ is exact and preserves injective objects, and therefore preserves injective resolutions.)

Now suppose $C$ is a cotorsion $\mathcal{O}_{p}$-module and $F$ is a flat $\mathcal{O}_{X}$-module. We wish to show that $S_{p}(C)$ is a cotorsion $\mathcal{O}_{X}$-module. But $F_{p}$ is a flat $\mathcal{O}_{p}$-module, so $\operatorname{Ext}^{1}\left(F, S_{p}(C)\right) \cong \operatorname{Ext}^{1}\left(F_{p}, C\right)=0$. Therefore $S_{p}(C)$ is cotorsion.

In order to verify the first hypothesis of Theorem 4.12 , we would like to find a large enough cardinal $\kappa$ so that the $\kappa$-generated $\mathcal{O}_{X}$-modules coincide with the $\kappa$-presentable $\mathcal{O}_{X}$-modules and so that these notions may be used in place of cardinality. Lemma 7.7 will allow us to do this. Our next lemma below however states that the category of $\mathcal{O}_{X}$-modules has a set of flat generators. This is a standard fact but we document it now since it will be used in the proof of Lemma 7.7 and is needed for Corollary 7.8.

Let $\mathcal{O}_{X}$ be a ringed space. For each open $U \subseteq X$, extend $\mathcal{O}_{\mid U}$ by 0 outside of $U$ to get a presheaf, denoted $\mathcal{O}_{U}$. Now sheafify to get an $\mathcal{O}_{X}$-module, which we will denote $j !\left(\mathcal{O}_{U}\right)$.

Lemma 7.4. Let $\mathcal{O}_{X}$ be a ringed space. Then $\operatorname{Hom}\left(j !\left(\mathcal{O}_{U}\right), G\right) \cong G(U)$ for any $\mathcal{O}_{X}$-module $G$ and each open set $U \subseteq X$. Furthermore, $\left\{j !\left(\mathcal{O}_{U}\right): U \subseteq X\right\}$ is a set of flat generators for $\mathcal{O}_{X}$-Mod.

Proof. One can prove without much difficulty that $\operatorname{Hom}\left(\mathcal{O}_{U}, G\right) \cong G(U)$. So by the universal property of sheafification we get $\operatorname{Hom}\left(j !\left(\mathcal{O}_{U}\right), G\right) \cong G(U)$. It follows at once that the set forms a generating set since the modules $j !\left(\mathcal{O}_{U}\right)$ "pick out points". Also, each $j !\left(\mathcal{O}_{U}\right)$ is flat since $\left[j !\left(\mathcal{O}_{U}\right)\right]_{p} \cong\left(\mathcal{O}_{U}\right)_{p}$, which equals $\mathcal{O}_{p}$ if $p \in U$ and 0 if $p \in X \backslash U$.

Definition 7.5. We define the cardinality of a presheaf (or sheaf), $F$, to be $|F|=$ $\left|\coprod_{U \subseteq X} F(U)\right|$ where $U \subseteq X$ ranges over all the open sets in $X$.

In Lemmas 7.6 and 7.7 we let $\mathcal{O}_{X}$ be our ringed space and let $\mathcal{U}$ represent the set of all open sets $U \subseteq X$. We note now that if $\beta$ is an infinite cardinal in which $\beta>\left|\mathcal{O}_{X}\right|$, then automatically $\beta>|\mathcal{U}|$, since $\mathcal{O}(U)$ is nonempty for each $U \subseteq X$.

Lemma 7.6. Let $\beta$ be an infinite cardinal such that $\beta>\max \left\{|X|,\left|\mathcal{O}_{X}\right|\right\}$. Now let $\kappa=2^{\beta}$. If $S$ is a presheaf of $\mathcal{O}_{X}$-modules and $|S|<\kappa$, then $\left|S^{+}\right|<\kappa$ where $S^{+}$ is the sheafification.

Proof. Clearly $\left|S_{p}\right|<\kappa$ for each point $p \in X$. So by the sheafification construction (see the proof of Proposition-Definition II.1.2 of [Har77]) one can easily see that for each open $U \subseteq X$ we have $\left|S^{+}(U)\right|<\kappa^{\beta}$. However, $\kappa^{\beta}=\left(2^{\beta}\right)^{\beta}=2^{\left(\beta^{2}\right)}=2^{\beta}=\kappa$, so $\left|S^{+}(U)\right|<\kappa$. Therefore $\left|S^{+}\right|=\left|\coprod_{U \subseteq X} S^{+}(U)\right|<\kappa$.

Next suppose we have a presheaf of $\mathcal{O}_{X}$-modules, $S$, and suppose we have a subset

$$
W \subseteq \coprod_{U \subseteq X} S(U)
$$


where again $U \subseteq X$ ranges over all the open sets in $X$. We set $W_{U}=W \cap S(U)$. The set $W$ generates a presheaf $S^{\prime}$ and it is given by

$$
S^{\prime}(U)=\sum_{V \supseteq \bigcup U} \sum_{w \in W_{V}} r_{V, U}([\mathcal{O}(V)] w) .
$$

That is, $S^{\prime}(U)$ consists of all finite sums of the form

$$
r_{V_{1}, U}\left(\rho_{1} w_{1}\right)+r_{V_{2}, U}\left(\rho_{2} w_{2}\right)+\cdots+r_{V_{n}, U}\left(\rho_{n} w_{n}\right)
$$

where

$$
V_{i} \supseteq U, w_{i} \in W_{V_{i}}, \rho_{i} \in \mathcal{O}\left(V_{i}\right)
$$

and $r_{V_{i}, U}: V_{i} \rightarrow U$ are the restriction maps. It is straightforward to see that $S^{\prime}$ is a presheaf and upon reflection it is clearly the smallest subpresheaf containing $W$. Furthermore, if $\kappa>\left|\mathcal{O}_{X}\right|$ is an infinite cardinal, and $\kappa>|W|$, then we will have $\kappa>\left|S^{\prime}\right|$. If $S$ was a sheaf of $\mathcal{O}_{X}$-modules to start with, then $\left(S^{\prime}\right)^{+}$is the $\mathcal{O}_{X}$-submodule generated by $W$ which we will denote $S_{W}$. In this case note that if $\kappa=2^{\beta}$ where $\beta>\max \left\{|X|,\left|\mathcal{O}_{X}\right|\right\}$, then by Lemma 7.6, $\kappa>\left|S_{W}\right|$ whenever $\kappa>|W|$.

Lemma 7.7. Let $\beta$ be an infinite cardinal such that $\beta>\max \left\{|X|,\left|\mathcal{O}_{X}\right|\right\}$. Now let $\kappa=2^{\beta}$. Also assume that $\kappa$ is large enough that each $j !\left(\mathcal{O}_{U}\right)$ is $\kappa$-generated. Then the following are equivalent for an $\mathcal{O}_{X}$-module $S$.

(1) $|S|<\kappa$.

(2) $S$ is к-generated.

(3) $S$ is $\kappa$-presentable.

Proof. (1) $\Rightarrow(2)$. We use Fact A.5. Suppose $|S|<\kappa$ and say $S=\sum_{i \in I} S_{i}$ is a $\kappa$-directed union of $\mathcal{O}_{X}$-submodules. Note that we will be done if we can show that for each open $U \subseteq X$ and each $x \in S(U)$, there exists an $i \in I$ such that $x \in S_{i}(U)$. Indeed if this were true, then by the large choice of $\kappa$ and the fact that the union is $\kappa$-filtered, we would take the union of all such $S_{i}(U)$ to display $S=S_{i}$ for some particular $i \in I$. (Note also that the assertion we wish to prove is not obvious since we must sheafify when taking the direct union.)

Now let $x \in S(U)$. From our choice of $\kappa$ we know that each $j !\left(\mathcal{O}_{U}\right)$ is $\kappa$ generated and so the canonical map $\operatorname{colim}_{i \in I} \operatorname{Hom}\left(j !\left(\mathcal{O}_{U}\right), S_{i}\right) \rightarrow \operatorname{Hom}\left(j !\left(\mathcal{O}_{U}\right), S\right)$ is an isomorphism. Now using Lemma 7.4 this translates to an isomorphism $\operatorname{colim}_{i \in I} S_{i}(U) \cong S(U)$. Through this isomorphism we see that $x \in S_{i}(U)$ for some $i \in I$.

(2) $\Rightarrow(1)$. Let $\mathcal{W}$ be the collection of all subsets $W \subseteq \bigsqcup_{U \subseteq X} S(U)$ (where $U$ ranges over all open subsets of $X$ ) which satisfy $|W|<\kappa$. For each $W \in \mathcal{W}$, let $S_{W}$ represent the $\mathcal{O}_{X}$-submodule generated by $W$. Then $\left|S_{W}\right|<\kappa$ by Lemma 7.6. Note that $(\mathcal{W}, \subseteq)$ is $\kappa$-filtered and in fact $S$ is the $\kappa$-filtered union $\sum_{W \in \mathcal{W}} S_{W}$. By Fact A.5, $S=S_{W}$ for some $W \in \mathcal{W}$. So $|S|<\kappa$.

$(3) \Rightarrow(2)$ is automatic. We now prove $(2) \Rightarrow(3)$, using that (1) iff (2). First we point out that the category of $\mathcal{O}_{X}$-modules is locally $\kappa$-generated. Indeed each $S$ can be expressed as the $\kappa$-filtered union $S=\sum_{W \in \mathcal{W}} S_{W}$ where each $S_{W}$ is $\kappa$ generated as in the last paragraph. Therefore, we may use the characterization of $\kappa$-presentable objects in Fact A.12. Suppose $S$ is $\kappa$-generated and $T \rightarrow S$ is an epimorphism with $T$ a $\kappa$-generated $\mathcal{O}_{X}$-module. Then $|T|<\kappa$. So of course $|\operatorname{ker}(T \rightarrow S)|<\kappa$, which means $\operatorname{ker}(T \rightarrow S)$ is $\kappa$-generated. This proves $S$ is $\kappa$-presentable. 
Finally we prove that the flat model structure on $\mathrm{Ch}\left(\mathcal{O}_{X}\right.$-Mod) comes from the Kaplansky class of flat $\mathcal{O}_{X}$-modules. The proof of Corollary 7.8 will again rely on a result of Enochs, et.al. which can be found in [EO01].

If $S \subseteq F$ is a subpresheaf (or subsheaf) we call $S$ presheaf pure if $S(U)$ is a pure $\mathcal{O}(U)$-submodule of $F(U)$ for each open $U$. We say $S \subseteq F$ is stalkwise pure if $S_{p}$ is a pure $\mathcal{O}_{p}$-submodule of $F_{p}$ for each $p \in X$.

Corollary 7.8. Let $\mathcal{G}=\mathcal{O}_{X}$-Mod where $\mathcal{O}_{X}$ is a sheaf of rings on a topological space $X$ and let $\mathcal{F}$ be the class of flat $\mathcal{O}_{X}$-modules. Then $\mathcal{F}$ is a Kaplansky class and it induces a model structure on $\mathrm{Ch}(\mathcal{G})$ we call the flat model structure. The cofibrations are the monomorphisms with dg-flat cokernels and the fibrations are the epimorphisms with dg-cotorsion kernels. Furthermore this model structure is cofibrantly generated and monoidal with respect to the usual tensor product of chain complexes.

Proof. Let $\beta$ be an infinite cardinal such that $\beta>\max \left\{|X|,\left|\mathcal{O}_{X}\right|\right\}$. Now let $\kappa=2^{\beta}$. Using Fact A.2 we can also assume that $\kappa$ is large enough that each $j !\left(\mathcal{O}_{U}\right)$ is $\kappa$ generated. We let $\mathcal{F}$ be the class of flat sheaves and claim that $\mathcal{F}$ is a $\kappa$-Kaplansky class.

So let $S^{\prime} \subseteq F \neq 0$ where $S^{\prime}$ is $\kappa$-generated and $F$ is flat. By Lemma 7.7, $\left|S^{\prime}\right|<\kappa$. With minor adjustments to the proof of Proposition 2.4 of [EO01] we can find an $\mathcal{O}_{X}$-submodule $S \subseteq F$ which is presheaf pure and such that $S^{\prime} \subseteq S$ and $|S|<\kappa$. It follows that $S \subseteq S^{+}$and $S^{+} \subseteq F$ is stalkwise pure. (This is not hard, but one could also see a proof in Lemma 4.7 of [Gil06].) It follows immediately that $S^{+}$and $F / S^{+}$ are flat. Furthermore, by Lemma 7.6 we have $\left|S^{+}\right|<\kappa$ and so by Lemma 7.7 we have that $S^{+}$is $\kappa$-presentable. This completes the proof that $\mathcal{F}$ is a $\kappa$-Kaplansky class.

Note that $\mathcal{O}_{X}$-Mod is locally $\kappa$-generated by the argument given in the last paragraph of the proof of Lemma 7.7. By our large choice of the cardinal $\kappa$ it follows that $\mathcal{O}_{X}$-Mod is also locally $\kappa$-presentable. So we are done verifying properties (1) and (2) of Theorem 4.12 .

It is a standard fact that $\mathcal{F}$ is closed under retracts, extensions and direct limits. So we focus on proving property (4). We let $Y$ be an exact dg-flat complex of sheaves. We want to show that it is a flat complex. I.e. that is has flat cycles in each degree. Note that we will be done if we can show that the "stalk complex" $Y_{p}$ is a flat complex of $\mathcal{O}_{p}$-modules for each $p \in X$. We know from the proof of Corollary 7.2 that an exact dg-flat complex of $\mathcal{O}_{p}$-modules is a flat complex. So we will simply show that $Y_{p}$ is a dg-flat complex. So let $Y_{p} \rightarrow C$ be a morphism where $C$ is a cotorsion complex of $\mathcal{O}_{p}$-modules. (This means that $C$ is exact and each cycle is cotorsion.) We want to show this map is null homotopic. But using the skyscraper functor and its adjoint property gives us a morphism $Y \rightarrow S_{p}(C)$ of complexes. Since $S_{p}(-)$ is exact and preserves cotorsion modules by lemma 7.3 we see that $S_{p}(C)$ is a cotorsion complex and by definition of dg-flat $Y \rightarrow S_{p}(C)$ is null homotopic. From this we can deduce that $Y_{p} \rightarrow C$ is also null homotopic.

Thus we have a cofibrantly generated model structure on $\mathrm{Ch}(\mathcal{G})$ where the cofibrations are the monomorphisms with cokernels in $d g \widetilde{\mathcal{F}}$ (dg-flat complexes) and the fibrations are the epimorphisms with kernels in $d g \widetilde{\mathcal{C}}$ (dg-cotorsion complexes).

We check now that the model structure is monoidal using Theorem 5.1. Again this is easy. The tensor product of two flat sheaves is also flat. The unit is the structure sheaf $\mathcal{O}_{X}$ which is flat too. 


\section{ApPendix A. LOCALly PRESENTABLE CATEGORIES}

Tibor Beke showed (Proposition 3.10 of [Bek00]) that Grothendieck categories are locally presentable. Here we gather some properties of locally presentable categories from the literature which will be needed for this paper. Virtually everything here can be found scattered throughout [AR94] and [Sten75].

Throughout this section, we will assume all cardinals are regular. These are infinite cardinals which are not the sum of a smaller number of smaller cardinals. For example, $\aleph_{\omega}=\sum_{n<\omega} \aleph_{n}$ is NOT a regular cardinal. All infinite successor cardinals are regular though.

Definition A.1. Let $\mathcal{C}$ be a category and let $\kappa$ be a regular cardinal.

A $\kappa$-filtered category is a category $\mathcal{K}$, for which every subcategory with less than $\kappa$ morphisms has a cocone. A $\kappa$-filtered diagram is simply a functor $F: \mathcal{K} \rightarrow \mathcal{C}$ in which $\mathcal{K}$ is a small $\kappa$-filtered category. By a $\kappa$-filtered colimit, we mean the colimit of a $\kappa$-filtered diagram.

An object $X$ in $\mathcal{C}$ is called $\kappa$-presentable if $\operatorname{Hom}_{\mathcal{C}}(X,-)$ preserves $\kappa$-filtered colimits. It is called presentable if it is $\kappa$-presentable for some regular cardinal $\kappa$.

An object $X$ in $\mathcal{C}$ is called $\kappa$-generated if $\operatorname{Hom}_{\mathcal{C}}(X,-)$ preserves $\kappa$-filtered colimits of monomorphisms. I.e., preserves the colimits of diagrams $F: \mathcal{K} \rightarrow \mathcal{C}$ for which $F(d): F(c) \rightarrow F\left(c^{\prime}\right)$ is a monomorphism for each $d: c \rightarrow c^{\prime}$ in $\mathcal{K}$. The object is called generated if it is $\kappa$-generated for some regular cardinal $\kappa$.

When $\mathcal{C}$ is the category of $R$-modules, the definitions above for $\kappa$-presentable and $\kappa$-generated agree with usual conventions. In fact, see below Fact A.12 and the example after Fact A.5.

As a special case we have the notion of a $\kappa$-directed poset which is really just a poset $(P, \leq)$ which is $\kappa$-filtered when thought of as a category. Then we have $\kappa$-directed colimits which are colimits of a functor $F:(P, \leq) \rightarrow \mathcal{K}$, where $(P, \leq)$ is a $\kappa$-directed poset. In a Grothendieck category $\mathcal{G}$, we often use the term $\kappa$-directed union. This is just a $\kappa$-directed colimit of a $\kappa$-directed set of subobjects, $\left\{S_{i}\right\}_{i \in I}$, of a given object $A$. It is denoted $\sum_{i \in I} S_{i}$ and it is a subobject of $A$ since direct limits are exact in a Grothendieck category.

Fact A.2. Let $\kappa^{\prime} \geq \kappa$ be regular cardinals. It is easy to see that any $\kappa^{\prime}$-filtered category is also a $\kappa$-filtered category. Thus any $\kappa^{\prime}$-filtered diagram is a $\kappa$-filtered diagram. Therefore, any $\kappa$-presentable (resp. generated) object is easily seen to be $\kappa^{\prime}$-presentable (resp. generated).

Fact A.3. Clearly, any $\kappa$-presentable object is $\kappa$-generated.

As explained in [AR94], $\kappa$-presentable and $\kappa$-generated objects can be defined in terms of $\kappa$-directed colimits instead of $\kappa$-filtered colimits. We also have the following facts which hold by generalizing the proofs in Chapter V.3 of [Sten75]. The proofs there are given for $\kappa=\omega$ (finitely generated objects), but everything carries over for an arbitrary $\kappa$.

Fact A.4. In a Grothendieck category, the image of a $\kappa$-generated object is again $\kappa$-generated. Also an extension of $\kappa$-generated objects is $\kappa$-generated.

Fact A.5. Let $\mathcal{G}$ be a Grothendieck category and $\kappa$ a regular cardinal. Then the following are equivalent for an object $X \in \mathcal{G}$ :

1) $X$ is $\kappa$-generated. 
2) Whenever $X=\sum_{i \in I} X_{i}$ is a $\kappa$-directed union of subobjects, we have $X=X_{i}$ for some $i \in I$.

3) Whenever $X=\sum_{i \in I} X_{i}$ is any union of subobjects, we have $X=\sum_{i \in J} X_{i}$ for some $J \subseteq I$ with $|J|<\kappa$.

Proof. The first two are equivalent by generalizing the proofs in Chapter V.3 of [Sten75]. The equivalence of the second two is not hard to prove but you need the fact that a colimit of less than $\kappa$-many $\kappa$-generated objects is again $\kappa$-generated.

Example. Let $R$ be a ring and $M$ an $R$-module. $M$ is $\kappa$-generated iff there exists a set $S \subseteq M$ with $|S|<\kappa$ such that $M=\sum_{x \in S} R x$.

Proof. $(\Rightarrow)$. Let $\mathcal{S}$ be the collection of all subsets $S \subseteq M$ such that $|S|<\kappa$. Let $M_{S}$ be the submodule $\sum_{x \in S} R x$. Note that $(\mathcal{S}, \subseteq)$ is $\kappa$-filtered and in fact $M$ is the $\kappa$-filtered union $\sum_{S \in \mathcal{S}} M_{S}$. Using Fact A.5, $M=M_{S}$ for some $S \in \mathcal{S}$.

$(\Leftarrow)$. Again we will use Fact A.5. Let $M=\sum_{i \in I} M_{i}$ be a $\kappa$-filtered union. By assumption, there is an $S \subseteq M$ such that $M=M_{S}(|S|<\kappa)$. For each $x \in S$, there corresponds some $i \in I$ such that $x \in M_{i}$. But $\sum_{i \in I} M_{i}$ is $\kappa$-filtered and $|S|<\kappa$, so there exists $i_{0} \in I$ such that $S \subseteq M_{i_{0}}$. So $M=M_{i_{0}}$.

Definition A.6. Any cocomplete category $\mathcal{C}$ is called locally $\kappa$-presentable if each object is a $\kappa$-filtered colimit of $\kappa$-presentable objects and the class of $\kappa$-presentable objects is essentially small, meaning there are only a sets worth of $\kappa$-presentable objects up to isomorphism. A category $\mathcal{C}$ is simply called locally presentable if it is locally $\kappa$-presentable for some regular cardinal $\kappa$.

Analogously, we define a locally $\kappa$-generated category as a cocomplete category in which each object is a $\kappa$-filtered union of $\kappa$-generated subobjects and the class of $\kappa$-generated objects is essentially small. A category $\mathcal{C}$ is simply called locally generated if it is locally $\kappa$-generated for some regular cardinal $\kappa$.

For example, if $R$ is a ring, then every $R$-module is an $\omega$-filtered union (usually just called filtered union) of its $\omega$-generated submodules (finitely generated submodules). So the category of $R$-modules is locally $\omega$-generated (locally finitely generated).

In general, as pointed out in [Sten75] (pp. 122), an arbitrary Grothendieck category may not even have any nonzero finitely generated objects. Such a category is clearly not locally finitely generated. However, the "Local Generation Theorem" ( [AR94], pp.54) says that a cocomplete category $\mathcal{C}$ is locally presentable if and only if it is locally generated. But be careful; this is NOT the same as saying $\mathcal{C}$ is locally $\kappa$-presentable if and only if it is locally $\kappa$-generated. For an example, see [AR94], Example 1.71, pp.56. Nevertheless, one direction does hold. For a proof of the following fact, see the first paragraph of the proof to the "Local Generation Theorem"( [AR94], pp.54).

Fact A.7. If $\mathcal{C}$ is locally $\kappa$-presentable, then $\mathcal{C}$ is locally $\kappa$-generated.

Fact A.8. Let $\kappa^{\prime} \geq \kappa$ be regular cardinals. Then $\mathcal{C}$ locally $\kappa$-presentable (resp. generated) implies $\mathcal{C}$ locally $\kappa^{\prime}$-presentable (resp. generated).

Fact A.9. If $\mathcal{C}$ is locally presentable, then for any regular cardinal $\lambda$, the class of $\lambda$ presentable objects is essentially small. Similarly, the class of $\lambda$-generated objects is essentially small([AR94], Corollary 1.69). Any fixed set of representatives of 
the $\lambda$-presentable objects is denoted $\operatorname{Pres}_{\lambda} \mathcal{C}$, and a set of representatives of the $\lambda$-generated objects is denoted by $\operatorname{Gen}_{\lambda} \mathcal{C}$.

Fact A.10. Every object of a locally presentable category is presentable (and hence generated).

Fact A.11. Let $X$ be an object in a locally presentable category. Then the class of subobjects of $X$ is in fact a set and the class of quotient objects of $X$ is also a set. In [AR94] this is expressed by saying locally presentable categories are wellpowered and cowellpowered, respectively.

The following gives a useful characterization of $\kappa$-presentable objects. Again, the proof is obtained by generalizing the proofs in Chapter V.3 of [Sten75].

Fact A.12. Let $\mathcal{G}$ be a locally $\kappa$-generated Grothendieck category. An object $X$ is $\kappa$-presentable if and only if $X$ is $\kappa$-generated and every epimorphism $C \rightarrow X$ with $C \kappa$-generated has a $\kappa$-generated kernel.

\section{APPENDIX B. $\kappa$-GENERATED OBJECTS FOR VERY LARGE $\kappa$}

Here we prove that in a Grothendieck category, there exists a regular cardinal $\kappa$ for which the class of $\kappa$-generated objects coincides with the class of $\kappa$-presentable objects. As a result, this class of objects (which has a small skeleton) satisfies the 2 out of 3 property for short exact sequences.

Lemma B.1. Let $R$ be a ring and $M$ an $R$-module. Let $\kappa$ be a regular cardinal such that $\kappa>|R|$. Then $M$ is $\kappa$-generated iff $|M|<\kappa$.

Proof. We use the characterization of $\kappa$-generated provided by Example A.

First, if $|M|<\kappa$, then $M$ is clearly $\kappa$-generated since we can take the generating set to be $M$ itself. Conversely, suppose $M$ is $\kappa$-generated so that there exists a set $S \subseteq M$ for which $M=\sum_{x \in S} R x$ and $|S|<\kappa$. Then $|M|=\left|\sum_{x \in S} R x\right| \leq$ $\left|\bigoplus_{x \in S} R x\right|<\kappa$.

Lemma B.2. Let $R$ be a ring and $M$ an $R$-module. Let $\kappa$ be a regular cardinal such that $\kappa>|R|$. Then $M$ is $\kappa$-generated iff $M$ is $\kappa$-presentable.

Proof. Of course $\kappa$-presentable objects are always $\kappa$-generated, so it remains to show that under the given hypothesis, $\kappa$-generated objects are $\kappa$-presentable. But this is easy using Lemma B.1 and the fact that $M$ is $\kappa$-presentable iff $M$ is $\kappa$ generated and every epimorphism $N \rightarrow M$ with $N \kappa$-generated, has a $\kappa$-generated kernel. Indeed suppose $M$ is $\kappa$-generated and $N \rightarrow M$ is an epimorphism with $N$ $\kappa$-generated. Then $|N|<\kappa$. So of course $|\operatorname{ker}(N \rightarrow M)|<\kappa$.

Next we can prove that the notion of $\kappa$-generated coincides with $\kappa$-presentable in any Grothendieck category if we take $\kappa$ to be large enough. In the proof we use the fact that an equivalence of categories preserves $\kappa$-generated and $\kappa$-presentable objects. Indeed if $F: \mathcal{A} \rightarrow \mathcal{B}$ is an equivalence, then there exists $G: \mathcal{B} \rightarrow \mathcal{A}$ such that $G F \cong 1_{\mathcal{A}}$ and $F G \cong 1_{\mathcal{B}}$. Furthermore, $F$ is then both a left and right adjoint of $G$ (and so each preserves colimits). So if $X \in \mathcal{A}$ is $\kappa$-presentable then the functor $\mathcal{A}(X, G(-))$ preserves $\kappa$-filtered colimits. But $\mathcal{A}(X, G(-)) \cong \mathcal{B}(F(X),-)$, so $F(X)$ must be $\kappa$-presentable. On the other hand, if $F(X)$ is $\kappa$-presentable, then $\mathcal{B}(F(X), F(-))$ preserves $\kappa$-filtered colimits. Therefore, $\mathcal{B}(F(X), F(-)) \cong$ 
$\mathcal{A}(G F(X),-)$ preserves $\kappa$-filtered colimits and $G F(X) \cong X$ must be $\kappa$-presentable. The same argument (but with $\kappa$-filtered colimits of monomorphisms) shows that an equivalence preserves $\kappa$-generated objects.

Proposition B.3. Let $\mathcal{G}$ be a Grothendieck category. Then there exists a regular cardinal $\kappa$ for which the $\kappa$-generated objects coincide with the $\kappa$-presentable objects.

Proof. By the Gabriel-Popescu Theorem $([\operatorname{Sten} 75]), \mathcal{G}$ is equivalent to a subcategory of $R$-Mod for some ring $R$. (The Gabriel-Popescu Theorem says more than this, but we don't need the whole statement.) Since an equivalence of categories preserves $\kappa$ generated and $\kappa$-presentable objects, the result follows from Lemma B.2 by choosing $\kappa$ to be a regular cardinal $\kappa>|R|$.

\section{REFERENCES}

[AR94] J. Adámek and J. Rosický, Locally presentable and accessible categories, Number 189 in London Mathematical Society Lecture Note Series, Cambridge University Press, 1994.

[Bek00] Tibor Beke, Sheafifiable homotopy model categories, Math. Proc. Camb. Phil. Soc. vol.129, no.3, 2000, pp.447-475.

[BBE01] L. Bican, R. El Bashir and E. Enochs, All modules have flat covers, Bull. London Math Soc., vol. 33, no. 4, 2001, pp. 385-390.

[Bor94] Francis Borceux, Handbook of Categorical Algebra. 1-3, Encyclopedia of Mathematics and its Applications 50-52. Cambridge University Press, Cambridge, 1994.

[Con00] Brian Conrad, Grothendieck dualilty and base change, Lecture Notes in Mathematics, Vol. 1750, Springer-Verlag, 2000.

[ET01] Paul C. Eklof and Jan Trlifaj, How to make Ext vanish, Bull. London Math Soc., vol. 33, no. 1, 2001, pp. 41-51.

[EEGO] E. Enochs, S. Estrada, J.R. García Rozas and L. Oyonarte, Flat covers in the category of quasi-coherent sheaves over the projective line, Comm. Algebra 32, 2004, pp.1497-1508.

[EE05] E. Enochs, S. Estrada, Relative homological algebra in the category of quasi-coherent sheaves, Advances in Mathematics vol. 194, no. 2, 2005, pp.284-295.

[EJ01] E. Enochs and O. Jenda, Relative homological algebra, De Gruyter Expositions in Mathematics no. 30, Walter De Gruyter, New York, 2001.

[ELR02] E. Enochs, J.A. López-Ramos, Kaplansky classes, Rend. Sem. Mat. Univ. Padova, Vol. 107, 2002.

[EO01] E. Enochs, L Oyonarte, Flat covers and cotorsion envelopes of sheaves, Proceedings of the American Mathematical Society vol. 130, no. 5, 2001, pp.1285-1292.

[Gil04] James Gillespie, The flat model structure on Ch(R), Trans. Amer. Math. Soc. vol. 356, no. 8, 2004, pp. 3369-3390.

[Gil06] James Gillespie, The flat model structure on complexes of sheaves, Trans. Amer. Math. Soc. vol. 358, no. 7, 2006, pp. 2855-2874.

[Har77] Robin Hartshorne, Algebraic Geometry, Grauate Texts in Mathematics vol. 52, SpringerVerlag, New York, 1977.

[Hov02] Mark Hovey, Cotorsion theories, model category structures, and representation theory, Mathematische Zeitschrift, vol. 241, 553-592, 2002.

[Hov99] Mark Hovey, Model categories, Mathematical Surveys and Monographs vol. 63, American Mathematical Society, 1999.

[HPS97] M. Hovey, J. Palmieri, and N. Strickland, Axiomatic stable homotopy theory, Mem. Amer. Math. Soc., Vol. 128, no. 610, 1997.

[Kap58] I. Kaplansky, Projective modules, Annals of Mathematics, vol. 68, no. 2, pp.372 - 377 , September 1958.

[Lam99] T.Y. Lam, Lectures on Modules and Rings, Graduate Texts in Mathematics vol. 189, Springer-Verlag, New York, 1999.

[Lan97] S. Lang, Algebra, Addison-Wesley, third edition, 1997.

[Lip] J. Lipman, Notes on derived categories and derived functors, preprint, www.math.purdue.edu/ ${ }^{\sim}$ lipman.

[Lit82] Shigeru Litaka, Algebraic Geometry, Graduate Texts in Mathemamtics vol. 76, SpringerVerlag, New York 1982. 
[May1] J.P. May, Picard groups, Grothendieck rings, and Burnside rings of a category, Advances in Mathemamtics, vol. 163, no. 1, 2001 pp.1-16.

[May3] J.P. May, The additivity of traces in triangulated categories, Advances in Mathemamtics, vol. 163, no. 1, 2001 pp.34-73.

[Mur] D. Murfet, Derived categories of quasi-coherent sheaves, online at www.therisingsea.org.

[Qui67] Daniel G. Quillen, Homotopical algebra, Lecture Notes in Mathematics no. 43, SpringerVerlag, 1967.

[SS00] S. Schwede, B.E. Shipley, Algebras and modules in monoidal model categories, Proc. London Math. Soc., vol. 80, no. 2, 2000, pp. 491-511.

[Spa88] N. Spaltenstein, Resolutions of unbounded complexes, Compos. Math., vol. 65, no. 2, 1988, pp. 121-154.

[Sten75] Bo Stenström, Rings of Quotients, Die Grundlehren der mathematischen Wissenschaften in Einzeldarstellungen Band 217, Springer-Verlag, New York 1975.

[AJL97] L. Alonso Tarrío, A. Jeremías López and J. Lipman, Local homology and cohomology of schemes, Ann. Sci. École Norm. Sup. (4), vol. 30, no. 1, 1997, pp.1-39.

[TT90] R.W. Thomason, Thomas Trobaugh, Appendix B of Higher algebraic K-theory of schemes and of derived categories, The Grothendieck Festschrift, Vol. III, pp.409-417, Progr. Math., 88,1990

[Wei94] Charles A. Weibel, An Introduction to Homological Algebra, Cambridge Studies in Advanced Mathematics vol. 38, Cambridge University Press, 1994.

4000 University Drive, Penn State Greater Allegheny, McKeesport, PA 15132-7698 E-mail address, Jim Gillespie: jrg21@psu.edu 